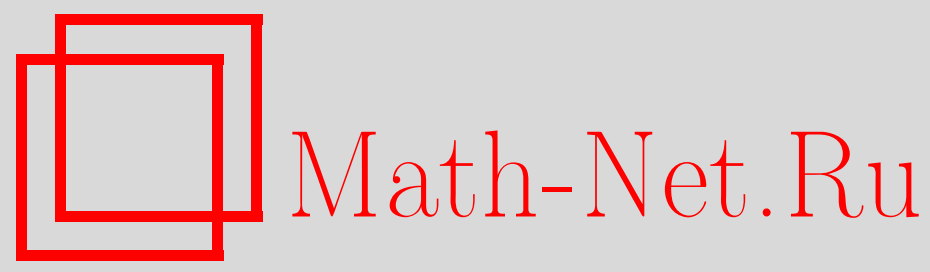

Г. В. Боков, Критерий конечной порожденности пропозициональных исчислений, Дискрет. матем., 2013, том 25, выпуск 3, 38-63

DOI: https://doi.org/10.4213/dm1247

Использование Общероссийского математического портала Math-Net.Ru подразумевает, что вы прочитали и согласны с пользовательским соглашением http://www . mathnet.ru/rus/agreement

Параметры загрузки:

IP : 54.198 .67 .100

26 апреля 2023 г., 13:00:18

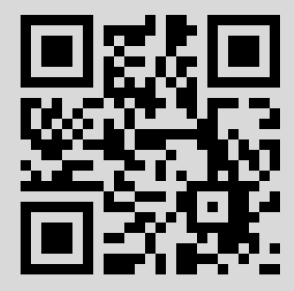




\title{
Критерий конечной порожденности пропозициональных исчислений
}

\author{
(c) 2013 г. . В. Боков
}

\begin{abstract}
В работе рассматриваются пропозициональные исчисления с произвольными модусными операциями вывода, аналогом которых является операция modus ponens. Для таких исчислений изучается вопрос о существовании и мощности систем образующих. Доказан критерий конечной порожденности пропозициональных исчислений с произвольными модусными операциями вывода. Для некоторых исчислений показано, что существование конечной полной системы тавтологий влечет за собой наличие базиса сколь угодно большой конечной мощности. Доказано существование пропозициональных исчислений со счетным базисом и без базиса, а также существование исчислений, в которых можно выделить полную подсистему, не имеющую базиса.
\end{abstract}

\section{Введение}

Особое внимание в изучении логических систем уделяется выделению систем образующих или базисов рассматриваемых систем. Системы образующих двузначных логик уже давно изучаются многими авторами. Так, Э. Пост [12] доказал, что если ограничиться только рассмотрением операции суперпозиции, то каждая двузначная логика конечно-порождена. При переходе от двузначных логик к $k$-значным для $k \geqslant 3$ обнаружились принципиально новые свойства этих объектов относительно выводимости или порождения одних формул другими. Так, для случая выводимости относительно суперпозиции [10] Мучник показал, что не каждая $k$-значная логика конечно-порождена, а Янов доказал, что для каждого $k \geqslant 3$ существует $k$-значная логика, не имеющая базиса.

Важным свойством классического исчисления высказываний [5] является существование конечного множества аксиом, из которых выводимы все тавтологии этого исчисления. Данное свойство называют конечной порожденностью исчисления. При расширении понятия исчисления обычно требуется, чтобы данное свойство сохранялось. Так в 1949 году Л. Хенкин [11] показал, что расширенный фрагмент исчисления высказываний, содержащий классическую импликацию, конечно-порожден относительной операции modus ponens: если выводимо $A$ и $A \rightarrow B$, то $B$ выводимо.

В данной работе вводится в рассмотрение пропозициональное исчисление с произвольными модусными операциями [6], доказываются необходимые и достаточные условия конечной порожденности такого исчисления и изучается вопрос о существовании и мощности базисов пропозициональных исчислений. 


\section{Основные понятия}

Множество натуральных чисел $\{1,2,3, \ldots\}$ обозначим через $\mathbb{N}$, а множество натуральных чисел с нулем $\mathbb{N} \cup\{0\}-$ через $\mathbb{N}_{+}$.

Пусть $E_{2}=\{0,1\}$. Через $P_{2}$ обозначим множество всех булевых функций [8]. Элементы множества $P_{2}$ будем обозначать строчными латинскими буквами $f, g, h, \ldots$, возможно, с индексами. Число существенных переменных функции $f \in P_{2}$ обозначим через $\varkappa(f)$. На множестве $P_{2}$ определим оператор замыкания [·], порожденный операцией суперпозиции [8]. Множество $Q \subseteq P_{2}$ замкнуто, если $[Q]=Q$.

Будем считать, что имеется некоторый счетный универсум переменных $\mathcal{U}$, элементы которого будем обозначать строчными латинскими буквами $x, y, z, \ldots$, возможно, с индексами. Если множество переменных $\mathcal{U}$ интерпретировать как переменные высказывания, принимающие только значения истина и ложь, то функции из $P_{2}$ можно рассматривать как логические связки над данными переменными.

Определим понятие формулы. Пусть $\Sigma \subseteq P_{2}-$ конечное множество функций и $X \subseteq \mathcal{U}$ - некоторое множество переменных; формулой над логическими связками из $\Sigma$ и множеством переменных $X$ назовем слово (конечную последовательность) в алфавите $\Sigma \cup X$, которое определяется следующим образом. Слово $x \in X$ является формулой. Если слова $\mathfrak{A}_{1}, \ldots, \mathfrak{A}_{n}-$ формулы и $f \in \Sigma-$ логическая связка арности $n$, то слово $f \mathfrak{A}_{1} \ldots \mathfrak{A}_{n}$ является формулой. Обозначим через $\Phi_{\Sigma}(X)$ множество всех формул над логическими связками из $\Sigma$ и множеством переменных $X$. Если $X=\mathcal{U}$, то множество $\Phi_{\Sigma}(X)$ будем для краткости обозначать через $\Phi_{\Sigma}$. Элементы множества $\Phi_{\Sigma}$ будем обозначать прописными готическими буквами $\mathfrak{A}, \mathfrak{B}, \mathfrak{C}, \ldots$, возможно, с индексами. Под равенством формул из $\Phi_{\Sigma}(X)$ будем понимать равенство соответствующих слов в алфавите $\Sigma \cup X$.

Будем говорить, что формула $\mathfrak{A} \in \Phi_{\Sigma}$ зависит от переменного $x \in \mathcal{U}$, если $x$ является подформулой в $\mathfrak{A}$. Зависимость формулы $\mathfrak{A}$ от переменной $x$ обозначим через $\mathfrak{A}(x)$, зависимость от переменных $x_{1}, \ldots, x_{n}$ - через $\mathfrak{A}\left(x_{1}, \ldots, x_{n}\right)$.

Каждой формуле $\mathfrak{F} \in \Phi_{\Sigma}$ однозначно сопоставим функцию из $[\Sigma \cup\{g(x)\}][8]$, где $g(x) \in P_{2}$ - тождественная функция. Поскольку каждую логическую связку из $\Sigma$ можно интерпретировать как соответствующую ей функцию, то по аналогии с определением формулы можно определить понятие интерпретации формуль. Каждая переменная задает тождественную функцию из $P_{2} ;$ если формулам $\mathfrak{A}_{1}, \ldots, \mathfrak{A}_{m}$ уже сопоставлены функции $f_{1}, \ldots, f_{m}$, то формуле $f \mathfrak{A}_{1} \ldots \mathfrak{A}_{m}$ сопоставим функцию $f\left(f_{1}, \ldots, f_{m}\right)$. Итак, каждой формуле $\mathfrak{F}$ из $\Phi_{\Sigma}$ можно сопоставить функцию из $P_{2}$, которую будем обозначать через $f_{\mathfrak{F}}$. В этом случае будем говорить, что формула $\mathfrak{F}$ выражает функцию $f_{\mathfrak{F}}$.

Формулу $\mathfrak{F}$ из $\Phi_{\Sigma}$ будем называть тавтологией или тождественно истинной, если $f_{\mathfrak{F}}\left(i_{1}, \ldots, i_{m}\right)=1$ при любых значениях $i_{1}, \ldots, i_{m}$ из $E_{2}$, где $m-$ это арность функции $f_{\mathfrak{F}}$. Обозначим через $\mathrm{Th}$ множество всех тавтологий в $\Phi_{\Sigma}$ :

$$
\mathrm{Th}=\left\{\mathfrak{F} \in \Phi_{\Sigma} \mid f_{\mathfrak{F}} \equiv 1\right\}
$$

На множестве формул $\Phi_{\Sigma}$ определим понятие модусной операции [6]. Пусть $\mathfrak{F}_{0}, \mathfrak{F}_{1}, \ldots, \mathfrak{F}_{m}-$ различные формулы из $\Phi_{\Sigma}\left(\left\{x_{1}, \ldots, x_{n}\right\}\right) ;$ набор $\left\langle\mathfrak{F}_{1}, \ldots, \mathfrak{F}_{m} ; \mathfrak{F}_{0}\right\rangle$ задает условную операцию $\omega: \Phi_{\Sigma}^{m} \rightarrow \Phi_{\Sigma}$, определенную следующим образом:

$$
\omega\left(\xi_{1}, \ldots, \xi_{m}\right)=\xi_{0} \Leftrightarrow \exists \mathfrak{A}_{1}, \ldots, \mathfrak{A}_{n} \in \Phi_{\Sigma}\left(\bigwedge_{i=0}^{m} \xi_{i}=\mathfrak{F}_{i}\left(\mathfrak{A}_{1}, \ldots, \mathfrak{A}_{n}\right)\right),
$$


где $\xi_{0}, \xi_{1}, \ldots, \xi_{m}$ - переменные, пробегающие множество формул $\Phi_{\Sigma}$. Следует отметить, что модусная операция является условной, поскольку в общем случае она может принимать разные значения для одного и того же набора значений переменных. Обозначим правую часть условия через $R_{\omega}\left(\xi_{0}, \xi_{1}, \ldots, \xi_{m}\right)$, тогда операцию $\omega$ можно записать в виде $\lambda$-функции [7]:

$$
\omega\left(\xi_{1}, \ldots, \xi_{m}\right)=\lambda_{\xi_{1}, \ldots, \xi_{m}}\left(\xi_{0} \mid R_{\omega}\left(\xi_{0}, \xi_{1}, \ldots, \xi_{m}\right)\right) .
$$

Следуя $[1,6]$, такие операции будем обозначать в виде схемы:

$$
\frac{\mathfrak{F}_{1}\left(x_{1}, \ldots, x_{n}\right), \ldots, \mathfrak{F}_{m}\left(x_{1}, \ldots, x_{n}\right)}{\mathfrak{F}_{0}\left(x_{1}, \ldots, x_{n}\right)} .
$$

Классическим примером модусной операции является операция modus ponens:

$$
\frac{x_{1}, x_{1} \rightarrow x_{2}}{x_{2}},
$$

которую обозначим через $\omega_{m p}$. Множество всех модусных операций на $\Phi_{\Sigma}$ обозначим через $\mathcal{M}_{\Sigma}$. Элементы множества $\mathcal{M}_{\Sigma}$ будем обозначать строчной греческой буквой $\omega$, а множества элементов из $\mathcal{M}_{\Sigma}$ - прописной греческой буквой $\Omega$, возможно, с индексами.

Нас будут интересовать не все модусные операции, а лишь те $\omega \in \mathcal{M}_{\Sigma}$, которые тавтологии переводят в тавтологии. Такие операции назовем допустимыми [4] на Th. Множество всех допустимых на Тh операций обозначим через $O_{\mathrm{Th}}$.

Кроме того, определим на множестве Тh операцию подстановки. Пусть $\mathfrak{A}$ - тавтология, содержащая переменное высказывание $x$, а $\mathfrak{B}$ - произвольная формула из $\Phi_{\Sigma}$. Тогда, если заменить в $\mathfrak{A}$ все вхождения $x$ на $\mathfrak{B}$, то полученная формула $\mathfrak{A}(\mathfrak{B})$ тоже будет тавтологией. В этом случае будем говорить, что из $\mathfrak{A}$ выводима $\mathfrak{A}(\mathfrak{B})$. Операцию будем записывать в виде схемы:

$$
\frac{\mathfrak{A}(x)}{\mathfrak{A}(y)} .
$$

Данную операцию можно опустить, если предполагать, что каждая тавтология задает не одну, а множество формул, получающихся из данной применением операции подстановки к всевозможным переменным данной тавтологии. Пользуясь свойством перестановочности операции подстановки относительно любой модусной операции, далее будем иногда опускать явное применение операции подстановки.

Расширим понятие операции подстановки на общий случай. Подстановкой будем называть всякое отображение $\sigma: \mathcal{U} \rightarrow \Phi_{\Sigma}$. Результатом применения подстановки $\sigma \mathrm{k}$ формуле $\mathfrak{A} \in \Phi_{\Sigma}$ является формула $\sigma \mathfrak{A}$, которая определяется по следующему правилу. Если $\mathfrak{A}=x \in \mathcal{U}$, то $\sigma \mathfrak{A}=\sigma(x)$, если же $\mathfrak{A}=f \mathfrak{B}_{1} \ldots \mathfrak{B}_{n}$, то $\sigma \mathfrak{A}=f \sigma \mathfrak{B}_{1} \ldots \sigma \mathfrak{B}_{n}$. Отличие подстановки как отображения $\sigma: \mathcal{U} \rightarrow \Phi_{\Sigma}$ от операции подстановки заключается в том, что $\sigma$ позволяет одновременно подставлять формулы вместо нескольких переменных. Множество всех подстановок $\sigma: X \rightarrow \Phi_{\Sigma}$, где $X \subseteq \mathcal{U}$, обозначим через $\Pi_{\Sigma}(X)$, а множество подстановок $\Pi_{\Sigma}(\mathcal{U})$ через $\Pi_{\Sigma}$. Ясно, что для любой подстановки $\sigma \in \Pi_{\Sigma}$ и каждой формулы $\mathfrak{A} \in \Phi_{\Sigma}$ формула $\sigma \mathfrak{A}$ есть результат многократного применения операции подстановки к формуле $\mathfrak{A}$.

Множество тавтологий Тh и множество допустимых операций $\Omega$ образуют алгебраическую систему $(\mathrm{Th}, \Omega)$, которую будем называть пропозициональным исчислением. Пусть $\Omega \subseteq O_{\text {Th }}-$ произвольное множество допустимых на $\mathrm{Th}$ модусных 
операций, тогда на Th можно определить оператор замыкания, порожденный операциями из $\Omega$ [3]. Этот оператор будем обозначать через $[\cdot]_{\Omega}$ или просто через [.], когда множество $\Omega$ фиксировано ${ }^{1}$.

Несложно проверить, что для любого множества модусных операций $\Omega \subseteq \mathcal{M}_{\Sigma}$ оператор $[\cdot]_{\Omega}$ является алгебраическим оператором замыкания [3].

Множество тавтологий $M \subseteq$ Th называется замкнутым относительно множества операций $\Omega$, если $[M]_{\Omega}=M$, и полным, если $[M]_{\Omega}=$ Th. Для произвольного $M \subseteq$ Th и $\mathfrak{A} \in \mathrm{Th}$ формулу $\mathfrak{A} \in[M]_{\Omega}$ назовем выводимой из множества формул $M$ и будем обозначать это через $M \vdash_{\Omega} \mathfrak{A}$ (или просто через $M \vdash \mathfrak{A}$, если множество $\Omega$ фиксировано). Множество тавтологий Тh будем называть конечно-порожденным относительно множества операций $\Omega$, если существует такое конечное множество $M \subseteq \mathrm{Th}$, что $[M]_{\Omega}=\mathrm{Th}$. Исчисление $(\mathrm{Th}, \Omega)$ конечно-порождено, если множество Th конечно-порождено относительно множества операций $\Omega$.

Для произвольного множества тавтологий $M \subseteq$ Th и произвольного множества

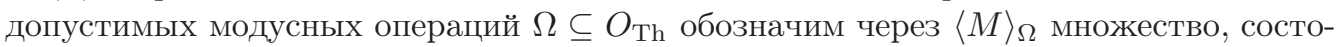
ящее из тавтологий множества $M$, из тавтологий $\mathfrak{A}$, для которых существует такая операция $\omega \in \Omega$ и такие формулы $\mathfrak{B}_{1}, \ldots, \mathfrak{B}_{m} \in M$, что $\omega\left(\mathfrak{B}_{1}, \ldots, \mathfrak{B}_{m}\right)=\mathfrak{A}$, а также из тавтологий $\mathfrak{A}$, для которых существуют такая формула $\mathfrak{B} \in M$ и подстановка $\sigma \in \Pi_{\Sigma}$, что $\mathfrak{A}=\sigma \mathfrak{B}$. Если множество операций $\Omega$ фиксировано, то будем писать $\langle M\rangle$ вместо $\langle M\rangle_{\Omega}$. Положим $\langle M\rangle_{\Omega}^{0}=M$ и $\langle M\rangle_{\Omega}^{l+1}=\left\langle\langle M\rangle_{\Omega}^{l}\right\rangle_{\Omega}$, тогда несложно убедиться, чTо

$$
[M]_{\Omega}=\bigcup_{l \geqslant 0}\langle M\rangle_{\Omega}^{l} .
$$

Параметр $l$ при этом играет роль длины вывода.

Каждую формулу из $\Phi_{\Sigma}$ можно представлять в виде ориентированного дерева, листьям которого приписаны переменные из $\mathcal{U}$, а внутренним вершинам - элементы множества $\Sigma$. Каждой позиции в этом дереве соответствует, с одной стороны, элемент множества $\Sigma \cup \mathcal{U}$, с другой - подформула из $\Phi_{\Sigma}$. Введем функции $\psi$ и $\eta$ для задания соответственно символа и подформулы данной формулы в данной позиции. Позиции в формуле будем задавать словами над множеством $\mathbb{N}$.

Функция $\psi$ сопоставляет каждой формуле $\mathfrak{F} \in \Phi_{\Sigma}$ отображение $\psi_{\mathfrak{F}}: \mathbb{N}^{*} \rightarrow \Sigma \cup$ $\mathcal{U} \cup\{\perp\}$, где $\perp-$ символ, не принадлежащий $\Sigma \cup \mathcal{U}$. Если $\mathfrak{F} \in \mathcal{U}$, то

$$
\psi_{\mathfrak{F}}(\alpha)=\left\{\begin{array}{l}
\mathfrak{F}, \text { если } \alpha=\Lambda, \\
\perp \text { в противном случае. }
\end{array}\right.
$$

Если $\mathfrak{F}=f \mathfrak{F}_{1} \ldots \mathfrak{F}_{k}$, то

$$
\psi_{\mathfrak{F}}(\alpha)=\left\{\begin{array}{c}
f, \text { если } \alpha=\Lambda, \\
\psi_{\mathfrak{F}_{i}}(\beta), \text { если } \alpha=i \beta, 1 \leqslant i \leqslant k, \\
\perp \text { в противном случае. }
\end{array}\right.
$$

Функция $\eta$ сопоставляет каждой формуле $\mathfrak{F} \in \Phi_{\Sigma}$ отображение $\eta_{\mathfrak{F}}: \mathbb{N}^{*} \rightarrow \Phi_{\Sigma} \cup\{\perp\}$. Если $\mathfrak{F} \in \mathcal{U}$, то

$$
\eta_{\mathfrak{F}}(\alpha)=\left\{\begin{array}{l}
\mathfrak{F}, \text { если } \alpha=\Lambda, \\
\perp \text { в противном случае. }
\end{array}\right.
$$

\footnotetext{
${ }^{1}$ Во избежание увеличения количества обозначений, операторы замыкания для множеств различной природы имеют одно и тоже обозначение [·].
} 
Если $\mathfrak{F}=f \mathfrak{F}_{1} \ldots \mathfrak{F}_{k}$, то

$$
\eta_{\mathfrak{F}}(\alpha)=\left\{\begin{array}{c}
\mathfrak{F}, \text { если } \alpha=\Lambda, \\
\eta_{\mathfrak{F}_{i}}(\beta), \text { если } \alpha=i \beta, 1 \leqslant i \leqslant k, \\
\perp \text { в противном случае. }
\end{array}\right.
$$

Несложно заметить, что отображения $\psi$ и $\eta$ связаны соотношением: для любой формулы $\mathfrak{F} \in \Phi_{\Sigma}$ и любого слова $\alpha \in \mathbb{N}^{*}$ выполнено $\psi_{\mathfrak{F}}(\alpha)=\psi_{\eta_{\mathfrak{F}}(\alpha)}(\Lambda)$.

\section{Критерий конечной порожденности}

Будем считать, что все логические символы из $\Sigma$ существенно зависят от всех своих переменных.

Пусть $X \subseteq \mathcal{U}$ - некоторое множество переменных; обозначим через $\mathcal{M}_{\Sigma}(X) \subseteq$ $\mathcal{M}_{\Sigma}$ множество всех операций над переменными из $X$ :

$$
\mathcal{M}_{\Sigma}(X)=\left\{\omega \in \mathcal{M}_{\Sigma} \mid \omega=\left\langle\mathfrak{F}_{1}, \ldots, \mathfrak{F}_{m} ; \mathfrak{F}_{0}\right\rangle \text { и } \mathfrak{F}_{i} \in \Phi_{\Sigma}(X), i=0,1, \ldots, m\right\} .
$$

Определим на множестве $\mathcal{M}_{\Sigma}$ операцию выводимости. Операция $\omega=$ $\left\langle\mathfrak{F}_{1}, \ldots, \mathfrak{F}_{m} ; \mathfrak{F}_{0}\right\rangle \in \mathcal{M}_{\Sigma}\left(\left\{x_{1}, \ldots, x_{n}\right\}\right)$ выводима из множества операций $\Omega \subseteq \mathcal{M}_{\Sigma}$, если существует такое конечное множество тавтологий $M \subseteq \mathrm{Th}$, что для любых формул $\mathfrak{A}_{1}, \ldots, \mathfrak{A}_{n}, \mathfrak{B}_{1}, \ldots, \mathfrak{B}_{m} \in \Phi_{\Sigma}$ из выполнения условия $\overline{\mathfrak{B}}_{i}=\mathfrak{F}_{i}\left(\mathfrak{A}_{1}, \ldots, \mathfrak{A}_{n}\right) \in \mathrm{Th}, i=$ $1, \ldots, m$, следует выводимость

$$
M, \mathfrak{B}_{1}, \ldots, \mathfrak{B}_{n} \vdash_{\Omega} \mathfrak{F}_{0}\left(\mathfrak{A}_{1}, \ldots, \mathfrak{A}_{n}\right) .
$$

Выводимость операции $\omega$ из множества операций $\Omega$ обозначим через $\Omega \vdash \omega$, а множество всех выводимых операций - через $[\Omega]=\left\{\omega \in \mathcal{M}_{\Sigma} \mid \Omega \vdash \omega\right\}$. Несложно проверить, что [.] является оператором замыкания на $\mathcal{M}_{\Sigma}$. Будем говорить, что из множества операций $\Omega \subseteq \mathcal{M}_{\Sigma}$ выводимы операции $\Omega^{\prime} \subseteq \mathcal{M}_{\Sigma}$, и писать $\Omega \vdash \Omega^{\prime}$, если $\Omega^{\prime} \subseteq[\Omega]$

Следуя [9], определим несколько стандартных классов булевых функций.

Определение 1. Функция $f\left(x_{1}, \ldots, x_{n}\right) \in P_{2}$ называется:

$$
\begin{gathered}
\alpha \text {-функцией, если } f(x, \ldots, x)=x, \\
\beta \text {-функцией, если } f(x, \ldots, x)=1, \\
\gamma \text {-функцией, если } f(x, \ldots, x)=0, \\
\delta \text {-функцией, если } f(x, \ldots, x)=\bar{x} .
\end{gathered}
$$

Для произвольного множества функций $\Sigma \subseteq P_{2}$ обозначим через $\Sigma_{\alpha}, \Sigma_{\beta}, \Sigma_{\gamma}, \Sigma_{\delta}$ соответственно множества всех $\alpha-, \beta-, \gamma-, \delta$-функций множества $\Sigma$.

Определение 2. Функция $f\left(x_{1}, \ldots, x_{n}\right) \in P_{2}$ называется линейной, если для нее имеет место соотношение

$$
f\left(x_{1}, \ldots, x_{n}\right)=c_{0}+c_{1} x_{1}+\ldots+c_{n} x_{n},
$$

где $c_{i} \in E_{2}, i=0,1, \ldots, n$, и сумма берется по модулю 2 .

Множество всех линейных функций в $P_{2}$ обозначим через $L$. 
Определение 3. Функция $f\left(x_{1}, \ldots, x_{n}\right) \in P_{2}$ называется монотонной, если для любых таких наборов $\widetilde{\alpha}, \widetilde{\beta} \in E_{2}^{n}$, что $\widetilde{\alpha} \leqslant \widetilde{\beta}$, имеет место соотношение

$$
f(\widetilde{\alpha}) \leqslant f(\widetilde{\beta}) .
$$

Множество всех монотонных функций в $P_{2}$ обозначим через $M$.

Определение 4. Функция $f\left(x_{1}, \ldots, x_{n}, y\right) \in P_{2}$ называется импликативной, если для нее выполнено соотношение

$$
f\left(\sigma_{1}, \ldots, \sigma_{n}, \sigma_{0}\right)=0 \Leftrightarrow \sigma_{0}<\sigma_{i}, i=1, \ldots, n,
$$

где $\sigma_{i} \in E_{2}$.

Множество всех импликативных функций в $P_{2}$ обозначим через $I$.

Для каждого из введенных классов функций определим множества допустимых операций.

Для произвольного $n \in \mathbb{N}$ через $\bar{n}$ будем обозначать множество $\{1, \ldots, n\}$. Обозначим через $S_{n}, n \in \mathbb{N}$, множество перестановок на множестве $\bar{n}$. Четной подстановкой на $\bar{n}$ будем называть такое отображение $\pi: \bar{n} \rightarrow \bar{n}$, что если $n$ четно, то $\left|\pi^{-1}(i)\right|$ четно для любого $i \in \bar{n}$, а если $n$ нечетно, то $\left|\pi^{-1}(i)\right|$ четно для любого $i \in \bar{n}$, кроме какого-то одного, для которого $\pi^{-1}(i)=\{i\}$. Множество всех четных подстановок на $\bar{n}$ обозначим через $\Pi_{n}$.

Рассмотрим произвольное множество линейных функций $\Sigma \subseteq L$. Определим множество операций $\Omega_{p v}(\Sigma)$ для перестановки переменных. Для каждой $f \in \Sigma$, для которой $\varkappa(f)=n$, и каждой перестановки $\pi \in S_{n}$ добавим в $\Omega_{p v}(\Sigma)$ операцию

$$
\frac{f x_{1} \ldots x_{n}}{f x_{\pi(1)} \ldots x_{\pi(n)}} .
$$

Для каждых $f, g \in \Sigma$, для которых $\varkappa(f)+\varkappa(g)-1=n$, и каждой перестановки $\pi \in S_{n}$ добавим в $\Omega_{p v}(\Sigma)$ операцию

$$
\frac{f g x_{1} \ldots x_{n}}{f g x_{\pi(1)} \ldots x_{\pi(n)}} .
$$

Для каждых $f, g, h \in \Sigma$, для которых $\varkappa(f)+\varkappa(g)+\varkappa(h)-2=n$, и каждой перестановки $\pi \in S_{n}$ добавим в $\Omega_{p v}(\Sigma)$ операцию

$$
\frac{f g h x_{1} \ldots x_{n}}{f g h x_{\pi(1)} \ldots x_{\pi(n)}} .
$$

Определим множество операций $\Omega_{p l}(\Sigma)$ для перестановки логических связок. Для каждых $f, g \in \Sigma$, для которых $\varkappa(f)+\varkappa(g)-1=n$, добавим в $\Omega_{p l}(\Sigma)$ операцию

$$
\frac{f g x_{1} \ldots x_{n}}{g f x_{1} \ldots x_{n}} .
$$

Для каждых $f, g, h \in \Sigma$, для которых $\varkappa(f)+\varkappa(g)+\varkappa(h)-2=n$, добавим в $\Omega_{p l}(\Sigma)$ операцию

$$
\frac{f g h x_{1} \ldots x_{n}}{f h g x_{1} \ldots x_{n}} .
$$


Множество $\Omega_{p v} \cup \Omega_{p l}$ всех операций перестановок обозначим через $\Omega_{p}$.

Определим множество операций $\Omega_{\alpha}(\Sigma)$ для преобразования $\alpha$-функций. Для каждой $f \in \Sigma_{\alpha}$, для которой $\varkappa(f)=n$, и каждой четной подстановки $\pi \in \Pi_{n}$, для которой существует такое $i \in \bar{n}$, что $\pi^{-1}(i)=\{i\}^{2}$, добавим в $\Omega_{\alpha}(\Sigma)$ операции

$$
\frac{f x_{\pi(1)} \ldots x_{\pi(n)}}{x_{i}} \text { и } \frac{x_{i}}{f x_{\pi(1)} \ldots x_{\pi(n)}} \text {. }
$$

Определим множество операций $\Omega_{\sigma}(\Sigma)$ для преобразования $\sigma$-функций, где $\sigma \in$ $\{\beta, \gamma, \delta\}$. Для каждых $f, g \in \Sigma_{\sigma}$, для которых $\varkappa(f)+\varkappa(g)-1=n$, и каждой четной подстановки $\pi \in \Pi_{n}$, для которой существует такое $i \in \bar{n}$, что $\pi^{-1}(i)=\{i\}^{3}$, добавим в $\Omega_{\sigma}(\Sigma)$ операции

$$
\frac{f g x_{\pi(1)} \ldots x_{\pi(n)}}{x_{i}} \text { и } \frac{x_{i}}{f g x_{\pi(1)} \ldots x_{\pi(n)}} .
$$

Положим $\Omega_{L}=\Omega_{p} \cup \Omega_{\alpha} \cup \Omega_{\beta} \cup \Omega_{\gamma} \cup \Omega_{\delta}$. Несложно убедиться, что каждая операция

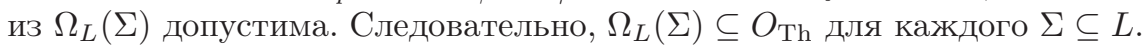

Монотонные функции в $P_{2}$ обладают тем свойством, что для них сокращенные дизъюнктивные нормальные формы (д.н.ф.) не содержат отрицаний [9]. Рассмотрим монотонную функцию $f\left(x_{1}, \ldots, x_{n}\right) \in M$; пусть

$$
\bigvee_{i=1}^{k} x_{i_{1}} \& \ldots \& x_{i_{n_{i}}}
$$

- ее сокращенная д.н.ф., где $x_{i_{j}} \in\left\{x_{1}, \ldots, x_{n}\right\}$. Всякий раз, когда переменные $x_{i_{1}}, \ldots, x_{i_{n_{i}}}$ принимают истинные значения, функция $f\left(x_{1}, \ldots, x_{n}\right)$ также принимает истинное значение. Основываясь на данном свойстве монотонных функций, для произвольного $\Sigma \subseteq M$ определим множество операций $\Omega_{M}(\Sigma)$. Для каждой функции $f\left(x_{1}, \ldots, x_{n}\right) \in \Sigma$ и для каждого конъюнкта $x_{i_{1}} \& \ldots \& x_{i_{k}}$ сокращенной д.н.ф. функции $f$ добавим в $\Omega_{M}$ операцию

$$
\frac{x_{i_{1}}, \ldots, x_{i_{k}}}{f x_{1} \ldots x_{n}} .
$$

Несложно убедиться, что каждая такая операция допустима. Следовательно,

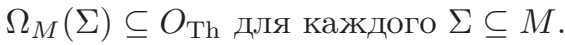

Для каждой импликативной функции $f \in[\Sigma] \cap I$ определим множество $\Omega_{I}(f)$, состоящее из операции

$$
\frac{x_{1}, \ldots, x_{n}, \mathfrak{F}\left(x_{1}, \ldots, x_{n}, y\right)}{y},
$$

где $\mathfrak{F} \in \Phi_{\Sigma}-$ формула, выражающая функцию $f$. Несложно убедиться, что такая

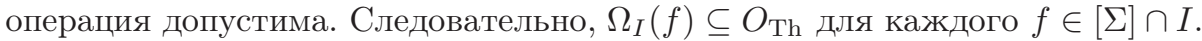

Для пропозициональных исчислений справедлив следующий критерий конечной порожденности.

Теорема 1 (Критерий конечно-порожденности). Для любого конечного множества логических связок $\Sigma \subseteq P_{2}$ и произвольного конечного множества допустимых на

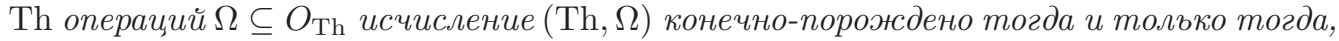
когда выполнено хотя бы одно из условий

\footnotetext{
${ }^{2}$ Такое $i$ найдется, поскольку для $\alpha$-функций $\varkappa(f)=2 k+1$ для некоторого $k \in \mathbb{N}_{+}$.

${ }^{3}$ Такое $i$ найдется, поскольку для $\sigma$-функций $\varkappa(f)+\varkappa(g)=2 k$ для некоторого $k \in \mathbb{N}_{+}$.
} 

1) $\Sigma \subseteq L, 1 \in[\Sigma]$ u $\Omega \vdash \Omega_{L}(\Sigma)$;
2) $\Sigma \subseteq M, 1 \in[\Sigma] u \Omega \vdash \Omega_{M}(\Sigma)$;
3) Найдется такая функиия $f \in[\Sigma] \cap I$, что $\Omega \vdash \Omega_{I}(f)$;
4) $\mathrm{Th}=\varnothing$.

\section{Мощность базисов}

Рассмотрим исчисление $(\mathrm{Th}, \Omega)$, где $\Omega$ - некоторое множество операций. Пусть $M \subseteq$ Th - замкнутый класс тавтологий, т.е. $[M]_{\Omega}=M$. Подмножество $\widetilde{M} \subseteq M$ множества $M$ будем называть базисом, если его замыкание $[\widetilde{M}]_{\Omega}$ совпадает со всем множеством $M$, а для любого его собственного подмножества $M^{\prime} \subset \widetilde{M}$ выполнено $\left[M^{\prime}\right]_{\Omega} \neq M$.

Следующая теорема показывает, что модусные операции обладают настолько богатыми выразительными способностями, что достаточно даже одной операции этого класса, чтобы получить весь спектр возможных базисов.

Теорема 2 (Мощности базисов). Для классического исчисления высказываний (Th, $\left.\left\{\omega_{m p}\right\}\right)$ с логическими связками $\Sigma=\{\wedge, \vee, \neg, \rightarrow\}$ выполнено каждое из условий

1. Существует конечно-порожденный замкнутый класс тавтологий, имеющий базис сколь угодно большой конечной мощности;

2. Существует замкнутый класс тавтологий, имеющий счетный базис;

3. Существует замкнутый класс тавтологий, не имеющий базиса;

4. Существует замкнутый класс тавтологий со счетным базисом, имеющий полную подсистему, из которой нельзя выделить базис.

\section{Доказательство утверждений}

\section{Выводимые операции и их свойства}

Следующая лемма показывает, что из допустимых операций выводимы только допустимые операции.

Лемма 1. Если $\Omega \subseteq O_{\mathrm{Th}}$, mo $[\Omega] \subseteq O_{\mathrm{Th}}$.

Доказательство. Пусть $\Omega \vdash \omega$, где $\omega=\left\langle\mathfrak{F}_{1}, \ldots, \mathfrak{F}_{m} ; \mathfrak{F}_{0}\right\rangle \in \mathcal{M}_{\Sigma}\left(\left\{x_{1}, \ldots, x_{n}\right\}\right)$, тогда существует такое конечное множество $M \subseteq \mathrm{Th}$, что для любых $\mathfrak{A}_{1}, \ldots, \mathfrak{A}_{n}, \mathfrak{B}_{0}, \mathfrak{B}_{1}, \ldots, \mathfrak{B}_{m} \in \Phi_{\Sigma}$ из выполнения условия $\mathfrak{B}_{i}=\mathfrak{F}_{i}\left(\mathfrak{A}_{1}, \ldots, \mathfrak{A}_{n}\right) \in \mathrm{Th}, i=$ $1, \ldots, m$, следует выводимость

$$
M, \mathfrak{B}_{1}, \ldots, \mathfrak{B}_{n} \vdash_{\Omega} \mathfrak{B}_{0},
$$

где $\mathfrak{B}_{0}=\mathfrak{F}_{0}\left(\mathfrak{A}_{1}, \ldots, \mathfrak{A}_{n}\right)$.

Так как $M \subseteq \mathrm{Th}, \mathfrak{B}_{i} \in \mathrm{Th}, i=1, \ldots, m$, и $\Omega \subseteq O_{\text {Тh }}$, то $\mathfrak{B}_{0} \in \mathrm{Th}$ и $\omega \in O_{\mathrm{Th}}$. Следовательно, $[\Omega] \subseteq O_{\text {Th }}$.

Лемма 2. Если исчисление $(\mathrm{Th}, \Omega)$ конечно-порождено, то $[\Omega]=O_{\mathrm{Th}}$. 
Доказательство. Так как $(\mathrm{Th}, \Omega)$ конечно-порождено, то существует конечное множество тавтологий $M \subseteq \mathrm{Th}$, для которого $[M]_{\Omega}=\mathrm{Th}$. Пусть $\omega \in O_{\mathrm{Th}}-$ произвольная допустимая операция, причем $\omega=\left\langle\mathfrak{F}_{1}, \ldots, \mathfrak{F}_{m} ; \mathfrak{F}_{0}\right\rangle$ и $\mathfrak{F}_{i} \in \Phi_{\Sigma}\left(\left\{x_{1}, \ldots, x_{n}\right\}\right), i=$ $0,1, \ldots, m$. Тогда для любых формул $\mathfrak{A}_{1}, \ldots, \mathfrak{A}_{n}, \mathfrak{B}_{0}, \mathfrak{B}_{1}, \ldots, \mathfrak{B}_{m} \in \Phi_{\Sigma}$, если $\mathfrak{B}_{i}=$ $\mathfrak{F}_{i}\left(\mathfrak{A}_{1}, \ldots, \mathfrak{A}_{n}\right) \in \mathrm{Th}, i=1, \ldots, m$, то $\mathfrak{B}_{0}=\mathfrak{F}_{0}\left(\mathfrak{A}_{1}, \ldots, \mathfrak{A}_{n}\right) \in \mathrm{Th}$. Поскольку $[M]_{\Omega}=\mathrm{Th}$, постольку $M \vdash_{\Omega} \mathfrak{B}_{0}$ и, следовательно,

$$
M, \mathfrak{B}_{1}, \ldots, \mathfrak{B}_{n} \vdash_{\Omega} \mathfrak{B}_{0} .
$$

Значит, $\Omega \vdash \omega$ и $[\Omega]=O_{\text {Th }}$.

Лемма 3. Если $\Omega, \Omega^{\prime} \subseteq O_{\mathrm{Th}}-$ конечные множества допустимых операций и $\Omega \vdash \Omega^{\prime}$, то из конечно-порожденности исчисления (Th, $\Omega^{\prime}$ ) следует конечнопорожденность исчисления (Th, $\Omega$ ).

Доказательство. Пусть $\Omega^{\prime}=\left\{\omega_{1}, \ldots, \omega_{k}\right\}$, где $\omega_{i}=\left\langle\mathfrak{F}_{1}^{i}, \ldots, \mathfrak{F}_{m_{i}}^{i} ; \mathfrak{F}_{0}^{i}\right\rangle$ и $\mathfrak{F}_{j}^{i} \in$ $\Phi_{\Sigma}\left(\left\{x_{1}, \ldots, x_{n_{i}}\right\}\right), i=1, \ldots, k, j=0,1, \ldots, m_{i}$. Так как для каждого $i=1, \ldots, k$ выполнено $\Omega \vdash \omega_{i}$, то существует такое конечное множество $M_{i} \subseteq \mathrm{Th}$, что для любых $\mathfrak{A}_{1}, \ldots, \mathfrak{A}_{n_{i}}, \mathfrak{B}_{0}, \mathfrak{B}_{1}, \ldots, \mathfrak{B}_{m_{i}} \in \Phi_{\Sigma}$ из выполнения условия $\mathfrak{B}_{j}=\mathfrak{F}_{j}^{i}\left(\mathfrak{A}_{1}, \ldots, \mathfrak{A}_{n_{i}}\right) \in$ $\mathrm{Th}, j=1, \ldots, m_{i}$, следует выводимость

$$
M_{i}, \mathfrak{B}_{1}, \ldots, \mathfrak{B}_{m_{i}} \vdash_{\Omega} \mathfrak{B}_{0},
$$

где $\mathfrak{B}_{0}=\mathfrak{F}_{0}^{i}\left(\mathfrak{A}_{1}, \ldots, \mathfrak{A}_{n_{i}}\right)$.

Тогда несложно показать, что для каждого $i=1, \ldots, k$ и любого множества тавтологий $Q \subseteq$ Тh верно включение

$$
[Q]_{\Omega \cup\left\{\omega_{i}\right\}} \subseteq\left[Q \cup M_{i}\right]_{\Omega}
$$

В самом деле, пусть $\mathfrak{A} \in[Q]_{\Omega \cup\left\{\omega_{i}\right\}}$. Рассмотрим наименьшее $l \geqslant 0$, для которого $\mathfrak{A} \in\langle Q\rangle_{\Omega \cup\left\{\omega_{i}\right\}}^{l}$ и $\mathfrak{A} \notin\langle Q\rangle_{\Omega \cup\left\{\omega_{i}\right\}}^{l-1}$. Докажем утверждение индукцией по длине вывода $l$. Если $l=0$, то $\mathfrak{A} \in Q \subseteq\left[Q \cup M_{i}\right]_{\Omega}$ и утверждение верно. Пусть $l>0$ и утверждение верно для всех $l^{\prime}<l$, т.е. $\langle Q\rangle_{\Omega \cup\left\{\omega_{i}\right\}}^{l-1} \subseteq\left[Q \cup M_{i}\right]_{\Omega}$. Если формула $\mathfrak{A} \in\langle Q\rangle_{\Omega \cup\left\{\omega_{i}\right\}}^{l}$ получена с помощью операций $\Omega$ или операции подстановки из формул множества $\langle Q\rangle_{\Omega \cup\left\{\omega_{i}\right\}}^{l-1}$, то $\mathfrak{A} \in\left[Q \cup M_{i}\right]_{\Omega}$ и утверждение верно. Если же $\mathfrak{A}$ получена с помощью операции $\omega_{i}$, то найдутся такие формулы $\mathfrak{B}_{1}, \ldots, \mathfrak{B}_{m_{i}} \in\langle Q\rangle_{\Omega \cup\left\{\omega_{i}\right\}}^{l-1}$, что $\omega_{i}\left(\mathfrak{B}_{1}, \ldots, \mathfrak{B}_{m_{i}}\right)=\mathfrak{A}$. Поскольку

$$
M_{i}, \mathfrak{B}_{1}, \ldots, \mathfrak{B}_{m_{i}} \vdash_{\Omega} \mathfrak{A},
$$

постольку $\mathfrak{A} \in\left[Q \cup M_{i}\right]_{\Omega}$, и утверждение доказано.

Если исчисление $\left(\mathrm{Th}, \Omega^{\prime}\right)$ конечно-порождено, то существует конечное множество тавтологий $M \subseteq \mathrm{Th}$, для которого $[M]_{\Omega^{\prime}}=$ Th. Если $M^{\prime}=M \cup \bigcup_{i=1}^{k} M_{i}$, то $\left|M^{\prime}\right|<\infty$ и верна цепочка включений:

$$
\mathrm{Th}=[M]_{\Omega^{\prime}} \subseteq[M]_{\Omega \cup \Omega^{\prime}} \subseteq\left[M^{\prime}\right]_{\Omega} \subseteq \mathrm{Th}
$$

Следовательно, $\left[M^{\prime}\right]_{\Omega}=\mathrm{Th}$ и исчисление $(\mathrm{Th}, \Omega)$ конечно-порождено. 


\section{Линейные пропозициональные исчисления}

Под линейными пропозициональными исчислениями будем понимать исчисления, множества формул которых образованы логическими связками из $L \subset P_{2}$. Обозначим через $\nu_{\mathfrak{A}}(a)$ число вхождений символа $x \in \Sigma \cup \mathcal{U}$ в формулу $\mathfrak{A}$. Переменное $x \in \mathcal{U}$ назовем значащим для формулы $\mathfrak{A}$, если $\nu_{\mathfrak{A}}(x)>0$.

Лемма 4. Для любой тавтологии $\mathfrak{A} \in \mathrm{Th}$ и для любого переменного $x \in \mathcal{U}$ число $\nu_{\mathfrak{A}}(x)$ четно.

Доказательство. В самом деле, каждой формуле $\mathfrak{A} \in \Phi_{\Sigma}$ однозначно соответствует линейная функция $f_{\mathfrak{A}} \in[\Sigma] \subseteq L$. По определению функция $f_{\mathfrak{A}}$ представима в виде

$$
f_{\mathfrak{A}}=c+k_{1} x_{1}+\ldots+k_{m} x_{m},
$$

где $c \in E_{2}, x_{1}, \ldots, x_{m}$ - все переменные, встречающиеся в формуле $\mathfrak{A}$, и $k_{i}=$ $\nu_{\mathfrak{A}}\left(x_{i}\right), i=1, \ldots, m$. Несложно убедиться, что каждое переменное $x \in \mathcal{U}$, для которого $\nu_{\mathfrak{A}}(x)=2 n+1, n \in \mathbb{N}_{+}$, является существенным ${ }^{4}$ для функции $f_{\mathfrak{A}}$. Поэтому, если $\mathfrak{A} \in \mathrm{Th}$, то функция $f_{\mathfrak{A}}$ не имеет существенных переменных и, следовательно, $\nu_{\mathfrak{A}}(x)=2 n, n \in \mathbb{N}$, для любого переменного $x$, встречающегося в формуле $\mathfrak{A}$. Для переменных $x \in \mathcal{U}$, которые не входят в $\mathfrak{A}$, выполнено $\nu_{\mathfrak{A}}(x)=0$. Лемма доказана.

Будем говорить, что формула $\mathfrak{A} \in \Phi_{\Sigma}$ получается из формулы $\mathfrak{B} \in \Phi_{\Sigma}$ перестановкой, и писать $\mathfrak{B} \rightsquigarrow \mathfrak{A}$, если $\mathfrak{A}$ можно вывести из $\mathfrak{B}$ применением только операций из множества $\Omega_{p}$.

Лемма 5. Для любых формул $\mathfrak{A}, \mathfrak{B} \in \Phi_{\Sigma}$ из $\mathfrak{A} \rightsquigarrow \mathfrak{B}$ следует, что $\mathfrak{B} \rightsquigarrow \mathfrak{A}$.

Доказательство. Все операции из множества $\Omega_{p}$ одноместны и обладают тем свойством, что для каждой из них существует обратная операция из этого же множества $\Omega_{p}$. Таким образом, $\Omega_{p}$ замкнута относительно добавления обратных отображений. Поэтому для любых формул $\mathfrak{A}, \mathfrak{B} \in \Phi_{\Sigma}$ и для любого вывода $\Gamma$ формулы $\mathfrak{B}$ из $\mathfrak{A}$, использующего только операции из множества $\Omega_{p}$, существует обратный вывод формулы $\mathfrak{A}$ из $\mathfrak{B}$, операции вывода которого являются обратными к операциям в $Г$ и, следовательно, принадлежат $\Omega_{p}$. Лемма доказана.

Тот факт, что $\mathfrak{A} \rightsquigarrow \mathfrak{B}$ и $\mathfrak{B} \rightsquigarrow \mathfrak{A}$, будем обозначать через $\mathfrak{A} \rightsquigarrow \mathfrak{B}$.

Сигнатурой формулы $\mathfrak{A} \in \Phi_{\Sigma}$ будем называть отображение $s_{\mathfrak{A}}: \Sigma \cup \mathcal{U} \rightarrow \mathbb{N}_{+}$, для которого $s_{\mathfrak{A}}(a)$ - это количество вхождений символа $a \in \Sigma \cup \mathcal{U}$ в формулу $\mathfrak{A}$. Следующая лемма показывает, как связаны между собой отношение њ и сигнатура формулы.

Лемма 6. Для любых бормул $\mathfrak{A}, \mathfrak{B} \in \Phi_{\Sigma}$ сигнатуры $s_{\mathfrak{A}}$ u $s_{\mathfrak{B}}$ совпадают, если $\mathfrak{A}$ un $\mathfrak{B}$.

Доказательство. Для каждой операции $\omega \in \Omega_{p}$, каждой формулы $\mathfrak{A} \in \Phi_{\Sigma}$ и любого символа $a \in \Sigma \cup \mathcal{U}$ имеем

$$
s_{\mathfrak{A}}(a)=s_{\omega(\mathfrak{A})}(a) .
$$

Если $\mathfrak{A}, \mathfrak{B} \in \Phi_{\Sigma}$ и $\mathfrak{A} \rightsquigarrow \mathfrak{B}$, то либо $\mathfrak{B}$ получена из $\mathfrak{A}$, либо $\mathfrak{A}$ получена из $\mathfrak{B}$ применением только операций из множества $\Omega_{p}$. Индукцией по длине вывода несложно показать, что для любого символа $a \in \Sigma \cup \mathcal{U}$ выполнено $s_{\mathfrak{A}}(a)=s_{\mathfrak{B}}(a)$. Лемма доказана.

\footnotetext{
43десь и далее предполагаем, что логические связки существенно зависят от всех своих переменных.
} 
Функциональный символ $f \in \Sigma$ имеет корневое вхождение в формулу $\mathfrak{A} \in \Phi_{\Sigma}$, если $\psi_{\mathfrak{A}}(\Lambda)=f$. Вхождение $\alpha \in \mathbb{N}^{*}$ символа $f \in \Sigma$ в формулу $\mathfrak{A} \in \Phi_{\Sigma}$ назовем сводимым, если для любого собственного непустого начала $\beta \in \mathbb{N}^{*}$ слова $\alpha$ выполнено $\psi_{\mathfrak{A}}(\beta)=g \in \Sigma$ и $\varkappa(g)>1$. Следующая лемма показывает, что некорневые сводимые вхождения функциональных символов в формулах линейного исчисления можно опустить ближе в корню дерева, представляющего эту формулу.

Лемма 7. Для любой формуль $\mathfrak{A} \in \Phi_{\Sigma}$ и любого некорневого сводимого вхождения функционального символа $f \in \Sigma$ в $\mathfrak{A}$ с $\varkappa(f) \geqslant 1$ найдется такая формула $\mathfrak{B} \in \Phi_{\Sigma}$, что $\mathfrak{A} \rightsquigarrow \mathfrak{B}, \psi_{\mathfrak{A}}(\Lambda)=\psi_{\mathfrak{B}}(\Lambda)$ u $\psi_{\mathfrak{B}}(k)=f$ для некоторого $k \in \mathbb{N}$.

Доказательство. Рассмотрим некорневое сводимое вхождение $\alpha \in \mathbb{N}^{*}$ символа $f$ в формулу $\mathfrak{A}$. Докажем утверждение леммы индукцией по длине слова $\alpha$. Поскольку $\alpha$ - некорневое вхождение, то $|\alpha| \geqslant 1$.

Если $|\alpha|=1$, то положим $\mathfrak{B}=\mathfrak{A}$. Тогда $\psi_{\mathfrak{A}}(\Lambda)=\psi_{\mathfrak{B}}(\Lambda)$ и $\psi_{\mathfrak{B}}(k)=f$, где $k=\alpha \in \mathbb{N}$.

Пусть утверждение верно для всех таких $\alpha \in \mathbb{N}^{*}$, что $1 \leqslant|\alpha|<l$. Докажем его для $\alpha$, имеющего длину $l$. Поскольку $|\alpha|>1$, найдутся такие $k, i \in \mathbb{N}$ и $\beta \in \mathbb{N}^{*}$, что $\alpha=k i \beta$. Рассмотрим отдельно два случая.

Если $|\beta|=0$, то формула $\mathfrak{A}$ представима в виде

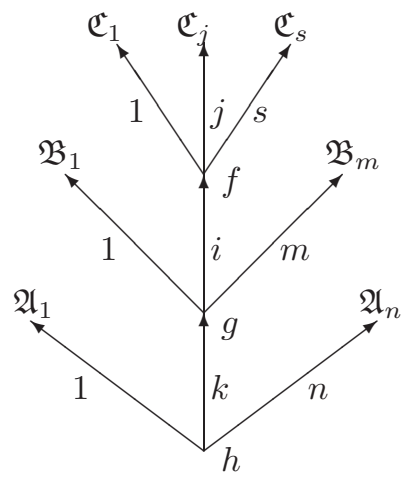

где $n, m$ и $s$ - арности функциональных символов $h, g$ и $f$ соответственно, $j \in \bar{s}$. Поскольку $s \geqslant 1$, такое $j$ всегда найдется. Применением операций $\Omega_{p}$ можно вывести формулу $\mathfrak{B}$ вида

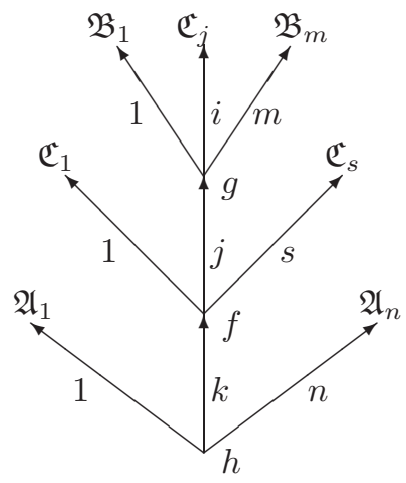


Ясно, что $\psi_{\mathfrak{B}}(\Lambda)=\psi_{\mathfrak{A}}(\Lambda)$ и $\psi_{\mathfrak{B}}(k)=f$, поэтому для случая $|\beta|=0$ утверждение леммы верно.

Если $|\beta|>0$, то найдутся такие $j \in \mathbb{N}$ и $\gamma \in \mathbb{N}^{*}$, что $\beta=j \gamma$ и $\alpha=k i j \gamma$. В этом случае формула $\mathfrak{A}$ представима в виде

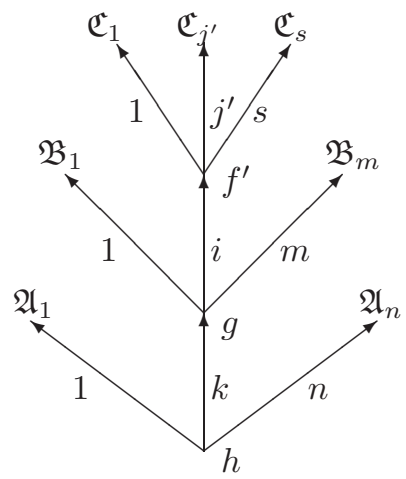

где $n, m$ и $s$ - арности функциональных символов $h, g$ и $f^{\prime}$ соответственно, $j^{\prime} \in$ $\bar{s} \backslash\{j\}$. Поскольку $s>1$, такое $j^{\prime}$ всегда найдется. Применением операций $\Omega_{p}$ можно вывести формулу $\mathfrak{C}$ вида

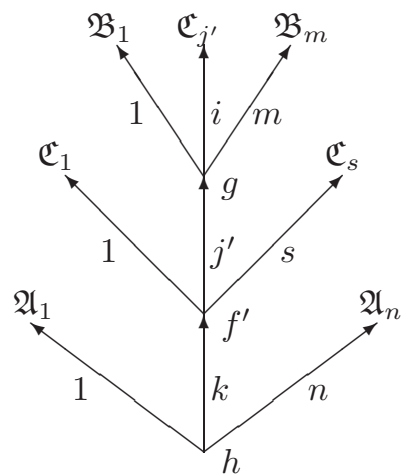

Для формулы $\mathfrak{C}$ имеем $\psi_{\mathfrak{A}}(\Lambda)=\psi_{\mathfrak{C}}(\Lambda)$ и $\psi_{\mathfrak{C}}(k j \gamma)=f$ и $|k j \gamma|=l-1$. По индуктивному предположению для формулы $\mathfrak{C}$ найдется такая формула $\mathfrak{B}$, что $\mathfrak{C} \rightsquigarrow \mathfrak{B}, \psi_{\mathfrak{C}}(\Lambda)=$ $\psi_{\mathfrak{B}}(\Lambda)$ и $\psi_{\mathfrak{B}}\left(k^{\prime}\right)=f$ для некоторого $k^{\prime} \in \mathbb{N}$. Так как $\mathfrak{A} \rightsquigarrow \mathfrak{C}$, то, пользуясь транзитивностью отношения «», получаем $\mathfrak{A} \rightsquigarrow \mathfrak{B}$, кроме того, $\psi_{\mathfrak{A}}(\Lambda)=\psi_{\mathfrak{C}}(\Lambda)$, поэтому $\psi_{\mathfrak{A}}(\Lambda)=\psi_{\mathfrak{B}}(\Lambda)$. Последнее доказывает утверждение леммы.

Следствие 1. Для любой формулы $\mathfrak{A} \in \Phi_{\Sigma}$ и любого некорневого сводимого вхождения функиионального символа $f \in \Sigma$ в $\mathfrak{A}$ c $\varkappa(f) \geqslant 1$ найдется такая формула $\mathfrak{B} \in \Phi_{\Sigma}$, что $\mathfrak{A} \longleftrightarrow \mathfrak{B} u \psi_{\mathfrak{B}}(\Lambda)=f$.

Доказательство. Согласно лемме 7 найдется такая формула $\mathfrak{C} \in \Phi_{\Sigma}$, что $\mathfrak{A} \rightsquigarrow \mathfrak{C}$, $\psi_{\mathfrak{A}}(\Lambda)=\psi_{\mathfrak{C}}(\Lambda)$ и $\psi_{\mathfrak{C}}(k)=f$ для некоторого $k \in \mathbb{N}$. Формулу $\mathfrak{C}$ можно представить в виде 


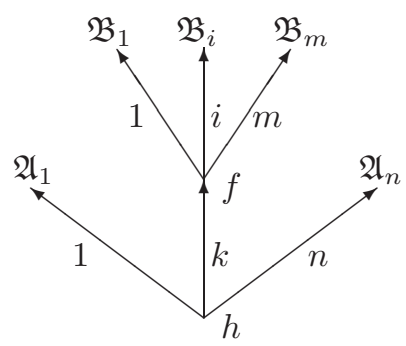

где $n$ и $m$ - арности функциональных символов $h$ и $f$ соответственно, $i \in \bar{m}$. Поскольку $m \geqslant 1$, такое $i$ всегда найдется. Применением операций $\Omega_{p}$ можно вывести формулу $\mathfrak{B}$ вида

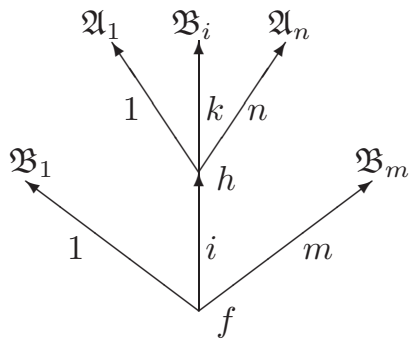

Ясно, что $\psi_{\mathfrak{B}}(\Lambda)=f$. Следствие доказано.

Будем называть эквивалентными и обозначать через $\mathfrak{A} \sim \mathfrak{B}$ формулы $\mathfrak{A}, \mathfrak{B} \in \Phi_{\Sigma}$, если $\mathfrak{A} \vdash \mathfrak{B}$ и $\mathfrak{B} \vdash \mathfrak{A}$.

Лемма 8. Для любой формуль $\mathfrak{A} \in \Phi_{\Sigma}$ существует такая формула $\mathfrak{B} \in \Phi_{\Sigma}$, что $\mathfrak{A} \sim \mathfrak{B}$ и выполнены условия

1) $\mathfrak{B}$ не содержит символа $f \in \Sigma_{\alpha}$, для которого $\varkappa(f)=1$;

2) $\mathfrak{B}$ содержит не более одного символа $f \in \Sigma_{\delta}$, для которого $\varkappa(f)=1$.

Доказательство. Обозначим число вхождений символов $f \in \Sigma_{\alpha}$ и символов $g \in \Sigma_{\delta}$, для которых $\varkappa(f)=1$ и $\varkappa(g)=1$, в формулу $\mathfrak{A}$ соответственно через $n_{\alpha}(\mathfrak{A})$ и $n_{\delta}(\mathfrak{A})$. Докажем утверждение леммы индукцией по $n_{\alpha}(\mathfrak{A})$ и $n_{\delta}(\mathfrak{A})$.

Если $n_{\alpha}(\mathfrak{A})=0$ и $n_{\delta}(\mathfrak{A}) \leqslant 1$, то формула $\mathfrak{A}$ удовлетворяет условиям 1$\left.), 2\right)$ и утверждение верно.

Пусть $n_{\alpha}(\mathfrak{A})>0$ или $n_{\delta}(\mathfrak{A})>1$ и утверждение леммы верно для всех формул $\mathfrak{B}$, у которых либо $n_{\alpha}(\mathfrak{B})<n_{\alpha}(\mathfrak{A})$, либо $n_{\delta}(\mathfrak{B})<n_{\delta}(\mathfrak{A})$. Докажем его для формулы $\mathfrak{A}$.

Рассмотрим наименьшее вхождение $\alpha \in \mathbb{N}^{*}$ символа $f \in \Sigma_{\alpha} \cup \Sigma_{\delta}$, для которого $\varkappa(f)=1$, в формулу $\mathfrak{A}$. Поскольку нет одноместных $\beta$-, $\gamma$-функций, существенно зависящих от одной переменной, то для любого собственного подслова $\beta \in \mathbb{N}^{*}$ слова $\alpha$ выполнено $\psi_{\mathfrak{A}}(\beta)=g$ и $\varkappa(g)>1$. Поэтому вхождение $\alpha$ символа $f$ является 
сводимым. По следствию 1 найдется такая формула $\mathfrak{B} \in \Phi_{\Sigma}$, что $\mathfrak{A}$ щ $\mathfrak{B}$ и $\psi_{\mathfrak{B}}(\Lambda)=$ $f$. Согласно лемме 6 для формулы $\mathfrak{B}$ выполнено $n_{\alpha}(\mathfrak{B})=n_{\alpha}(\mathfrak{A})$ и $n_{\delta}(\mathfrak{B})=n_{\delta}(\mathfrak{A})$. Возможны два случая.

Если $f \in \Sigma_{\alpha}$, то, применяя операцию из $\Omega_{\alpha}(\Sigma)$, заданную схемой

$$
\frac{f x}{x}
$$

к формуле $\mathfrak{B}$, получим, что $\mathfrak{A} \sim \mathfrak{C}$, где $\mathfrak{C}=\eta_{\mathfrak{B}}(1)$. Для формулы $\mathfrak{C}$ имеем $n_{\alpha}(\mathfrak{C})<$ $n_{\alpha}(\mathfrak{A})$ и $n_{\delta}(\mathfrak{C})=n_{\delta}(\mathfrak{A})$.

Если же $f \in \Sigma_{\delta}$, то рассмотрим некорневое сводимое вхождение $\beta \in \mathbb{N}^{*}$ символа $g \in \Sigma_{\alpha} \cup \Sigma_{\delta}, \varkappa(g)=1$, в формулу $\mathfrak{B}$. Поскольку $n_{\delta}(\mathfrak{B})>1$, такое вхождение всегда найдется. По лемме 7 найдется такая формула $\mathfrak{C} \in \Phi_{\Sigma}$, что $\mathfrak{B} \rightsquigarrow \mathfrak{C}, \psi_{\mathfrak{B}}(\Lambda)=\psi_{\mathfrak{C}}(\Lambda)$ и $\psi_{\mathfrak{C}}(k)=g$ для некоторого $k \in \mathbb{N}$. Тогда формула $\mathfrak{C}$ имеет вид $f g \mathfrak{D}$ для некоторой формулы $\mathfrak{D} \in \Phi_{\Sigma}$. Согласно лемме 6 для формулы $\mathfrak{C}$ выполнено $n_{\alpha}(\mathfrak{C})=n_{\alpha}(\mathfrak{A})$ и $n_{\delta}(\mathfrak{C})=n_{\delta}(\mathfrak{A})$.

Если $g \in \Sigma_{\alpha}$, то с помощью опереции перестановки связок из $\mathfrak{C}$ выводима формула $g f \mathfrak{D}$. Рассмотрение последней сводится к предыдущему случаю. Если же $g \in \Sigma_{\delta}$, то, применяя операцию из $\Omega_{\delta}(\Sigma)$, заданную схемой

$$
\frac{f g x}{x}
$$

к формуле $\mathfrak{C}$, получим, что $\mathfrak{C} \sim \mathfrak{D}$. Для формулы $\mathfrak{D}$ имеем $n_{\alpha}(\mathfrak{D})=n_{\alpha}(\mathfrak{A})$ и $n_{\delta}(\mathfrak{D})<$ $n_{\delta}(\mathfrak{A})$.

Во всех случаях для формулы $\mathfrak{A}$ найдется такая формула $\mathfrak{B}$, что $\mathfrak{A} \sim \mathfrak{B}$ и либо $n_{\alpha}(\mathfrak{B})<n_{\alpha}(\mathfrak{A})$, либо $n_{\delta}(\mathfrak{B})<n_{\delta}(\mathfrak{A})$. По предположению индукции утверждение леммы верно для формулы $\mathfrak{B}$, следовательно, оно верно и для формулы $\mathfrak{A}$. Лемма доказана.

Лемма 9. Для любой формуль $\mathfrak{A} \in \Phi_{\Sigma}$ существует такая формула $\mathfrak{B} \in \Phi_{\Sigma}$, что $\mathfrak{A} \sim \mathfrak{B}$ и $\mathfrak{B}$ не содержит символов $f \in \Sigma_{\alpha}$.

Доказательство. Докажем утверждение индукцией по числу $l$ вхождений символов $f \in \Sigma_{\alpha}$ в формулу $\mathfrak{A}$.

Если $l=0$, то $\mathfrak{A}$ удовлетворяет условиям и лемма доказана.

Пусть утверждение верно для всех $0 \leqslant l^{\prime}<l$, докажем его для $l$. Поскольку $l>0$, найдется вхождение символа $f \in \Sigma_{\alpha}$ в формулу $\mathfrak{A}$. Согласно лемме 8 можно считать, что $\mathfrak{A}$ не содержит одноместных символов из $\Sigma_{\alpha}$ и содержит не более одного символа из $\Sigma_{\delta}$. Без ограничения общности будем считать, что $\Sigma$ содержит только одну 0 -местную $\beta$-функцию и одну 0 -местную $\gamma$-функцию, которые обозначим через 1 и 0 соответственно. Возможны два случая.

Если $\mathfrak{A}$ не содержит одноместных символов из $\Sigma_{\delta}$, то по следствию 1 найдется такая формула $\mathfrak{B}$, что $\mathfrak{A} \rightsquigarrow \mathfrak{B}$ и $\mathfrak{B}=f \mathfrak{B}_{1} \ldots \mathfrak{B}_{n}$, где $n-$ арность символа $f$ и $n>$ 1. Поскольку $\mathfrak{B}$ не имеет одноместных связок, то последовательным применением операций $\Omega_{p}$ из $\mathfrak{B}$ можно вывести формулу $\mathfrak{C}$ вида

$$
f \underbrace{x_{1} \ldots x_{1}}_{n_{1}} \underbrace{x_{2} \ldots x_{2}}_{n_{2}} \ldots \underbrace{x_{k} \ldots x_{k}}_{n_{k}} \underbrace{1 \ldots 1}_{n_{k+1}} \underbrace{0 \ldots 0}_{n_{k+2}} \mathfrak{D},
$$


где $\left\{x_{1}, \ldots, x_{k}\right\}$ - все значащие переменные формулы $\mathfrak{A}, n_{1} \in \mathbb{N}, n_{i} \in \mathbb{N}_{+}, i=$ $2, \ldots, k+2$, и $\mathfrak{D}-$ это формула вида $g \mathfrak{D}_{1} \ldots \mathfrak{D}_{m}$, где $m$ - арность $g$ и

$$
\mathfrak{D}_{1}=\left\{\begin{array}{l}
x_{i}, \text { если } n_{i-1}=\nu_{\mathfrak{A}}\left(x_{i-1}\right) \text { и } 0 \leqslant n_{i}<\nu_{\mathfrak{A}}\left(x_{i}\right), \\
1, \text { если } n_{k}=\nu_{\mathfrak{A}}\left(x_{k}\right) \text { и } 0 \leqslant n_{k+1}<\nu_{\mathfrak{A}}(1), \\
0, \text { если } n_{k+1}=\nu_{\mathfrak{A}}(1) .
\end{array}\right.
$$

Для каждого $i \in \overline{k+1}$ в (1) $n_{i+1}=0$, если $n_{i}=0$, и $n_{i}=\nu_{\mathfrak{A}}\left(x_{i}\right)$, если $n_{i+1} \neq 0$. По сути $\mathfrak{B}$ в этом случае представляет собой дерево, внутренние вершины которого имеют хотя бы два выходных ребра, а операции $\Omega_{p}$ перебалансируют это дерево.

Если $\mathfrak{A}$ содержит одноместный символ $h \in \Sigma_{\delta}$, то по лемме 7 и следствию 1 найдется такая формула $\mathfrak{B}$, что $\mathfrak{A} \leadsto \mathfrak{B}$ и $\mathfrak{B}=h f \mathfrak{B}_{1} \ldots \mathfrak{B}_{n}$, где $n-$ арность символа $f$ и $n>1$. Поскольку $\mathfrak{B}_{i}$ не имеют одноместных связок, то последовательным применением операций $\Omega_{p}$ из $\mathfrak{B}$ можно вывести формулу $\mathfrak{C}$ вида

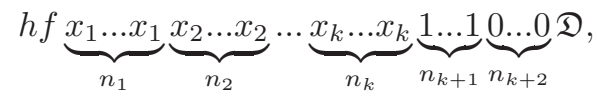

в которой используются те же обозначения, что в (1). Из этой формулы с помощью операций $\Omega_{p}$ получим формулу

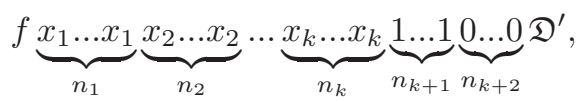

где $\mathfrak{D}^{\prime}=g \mathfrak{D}_{1} \ldots \mathfrak{D}_{m-1} \mathfrak{D}_{m}^{\prime}$ и $\mathfrak{D}_{m}^{\prime}=h \mathfrak{D}_{m}$.

В обоих случаях найдется формула $\mathfrak{B}$ вида $(1)$, для которой $\mathfrak{A} \rightsquigarrow \mathfrak{B}$.

Если $n_{i} \neq 0$ и $n_{i+1}=0$, где $i \leqslant k+1$, то $n_{1}, \ldots, n_{i-1}$ четны по лемме 4 . Так как $f \in \Sigma_{\alpha}$, то $n$ нечетно, поэтому $n_{i}$ тоже четно. Применяя операцию из $\Omega_{\alpha}$, заданную схемой

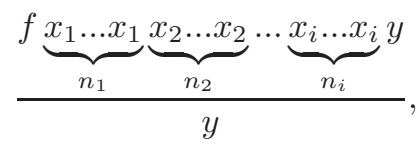

получим, что $\mathfrak{A} \sim \mathfrak{D}$.

Если $n_{k+1}$ четно, то $n_{1}, \ldots, n_{k}$ четны по лемме 4 . Следовательно, $n_{k+2}$ четно. Применяя операцию из $\Omega_{\alpha}$, заданную схемой

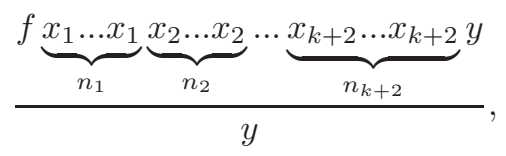

получим, что $\mathfrak{A} \sim \mathfrak{D}$.

Если $n_{k+1}$ нечетно, то $n_{k+2} \neq 0$ и $\mathfrak{D}_{1}=0$, поэтому, применяя операции из $\Omega_{p}$, имеем

$$
\mathfrak{A} \leadsto f \underbrace{x_{1} \ldots x_{1}}_{n_{1}} \underbrace{x_{2} \ldots x_{2}}_{n_{2}} \cdots \underbrace{x_{k} \ldots x_{k}}_{n_{k}} \underbrace{1 \ldots 1}_{n_{k+1}-1} \underbrace{0 \ldots 0}_{n_{k+2}+1} \mathfrak{D}^{\prime},
$$

где $\mathfrak{D}^{\prime}=g \mathfrak{D}_{1}^{\prime} \mathfrak{D}_{2} \ldots \mathfrak{D}_{m}$ и $\mathfrak{D}_{1}^{\prime}=1$. Отсюда, применяя операции $\Omega_{\alpha}$, можно вывести формулу $\mathfrak{D}^{\prime}$, причем $\mathfrak{A} \sim \mathfrak{D}^{\prime}$.

В любом случае найдется такая формула $\mathfrak{D} \in \Phi_{\Sigma}$, что $\mathfrak{A} \sim \mathfrak{D}$ и число вхождений символов $f \in \Sigma_{\alpha}$ в $\mathfrak{D}$ на единицу меньше, чем в $\mathfrak{A}$. По предположению индукции утверждение леммы верно для формулы $\mathfrak{D}$, следовательно, оно верно и для формулы $\mathfrak{A}$. Лемма доказана. 
Лемма 10. Для любой формулы $\mathfrak{A} \in \Phi_{\Sigma}$ существует такая формула $\mathfrak{B} \in \Phi_{\Sigma}$, что $\mathfrak{A} \sim \mathfrak{B}$ и $\mathfrak{B}$ содержит не более одного символа $f \in \Sigma_{\sigma}$, где $\sigma \in\{\beta, \gamma, \delta\}$.

Доказательство. Утверждение леммы будем доказывать индукцией по числу вхождений $n_{\mathfrak{A}}(\sigma)$ в формулу $\mathfrak{A}$ символов $f \in \Sigma_{\sigma}$, где $\sigma \in\{\beta, \gamma, \delta\}$. Если $n_{\mathfrak{A}}(\sigma) \leqslant 1$ для всех $\sigma \in\{\beta, \gamma, \delta\}$, то в качестве формулы $\mathfrak{B}$ можно взять $\mathfrak{A}$.

Пусть утверждение леммы верно для такого $n_{\mathfrak{A}}(\sigma)$, что $1 \leqslant n_{\mathfrak{A}}(\sigma)<k_{\sigma}$. Покажем, что оно верно и для $n_{\mathfrak{A}}(\sigma)=k_{\sigma}$.

Согласно лемме 8 можно считать, что $\mathfrak{A}$ не содержит одноместных символов из $\Sigma_{\alpha}$ и содержит не более одного символа из $\Sigma_{\delta}$. По аналогии с леммой 9 будем считать, что $\mathfrak{A}$ не содержит одноместных символов из $\Sigma_{\delta}$ и $\Sigma$ содержит только одну 0 -местную $\beta$-функцию и одну 0 -местную $\gamma$-функцию, которые также обозначим через 1 и 0 соответственно.

Поскольку $k_{\sigma}>1$, найдутся вхождения символов $f, g \in \Sigma_{\sigma}$ в формулу $\mathfrak{A}$. Тогда по лемме 7 и следствию 1 найдется такая формула $\mathfrak{B}$, что $\mathfrak{A} \leadsto \mathfrak{B}$ и $\mathfrak{B}=f g \mathfrak{B}_{1} \ldots \mathfrak{B}_{n}$, где $n=\varkappa(f)+\varkappa(g)-1$ и $n>1$. Так как $\mathfrak{B}$ не имеет одноместных связок, то последовательным применением операций $\Omega_{p}$ из $\mathfrak{B}$ можно вывести формулу $\mathfrak{C}$ вида

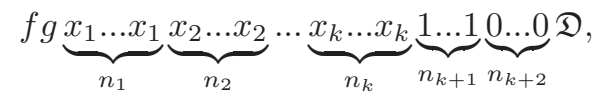

в которой используются те же обозначения, что в (1).

Если воспользоваться техникой доказательства леммы 9 и заменить всюду операции $\Omega_{\alpha}$ на операции $\Omega_{\sigma}$, то несложно убедиться, что найдется такая формула $\mathfrak{D} \in \Phi_{\Sigma}$, что $\mathfrak{A} \sim \mathfrak{D}$ и число вхождений символов $f \in \Sigma_{\sigma}$ в $\mathfrak{D}$ на единицу меньше, чем в $\mathfrak{A}$. По предположению индукции утверждение леммы верно для формулы $\mathfrak{D}$, следовательно, оно верно и для формулы $\mathfrak{A}$. Лемма доказана.

Для линейных пропозициональных исчислений выполнено следующее достаточное условие конечной порожденности.

Лемма 11. Если $\Sigma \subseteq L,|\Sigma|<\infty, 1 \in[\Sigma] u \Omega \subseteq O_{\mathrm{Th}},|\Omega|<\infty, \Omega \vdash \Omega_{L}(\Sigma)$, mo исчисление $(\mathrm{Th}, \Omega)$ конечно-порождено.

Доказательство. Согласно лемме 3 достаточно показать, что исчисление $\left(\mathrm{Th}, \Omega_{L}(\Sigma)\right)$ конечно-порождено. Пусть $\mathfrak{A}-$ произвольная тавтология из Тh. Согласно леммам 9 и 10 существует такая формула $\mathfrak{B} \in \Phi_{\Sigma}$, что $\mathfrak{A} \sim \mathfrak{B}$, причем $\mathfrak{B}$ не содержит символов $f \in \Sigma_{\alpha}$ и содержит не более одного символа $f \in \Sigma_{\sigma}$, где $\sigma \in\{\beta, \gamma, \delta\}$. Так как $\Omega_{L}(\Sigma) \subseteq O_{\text {Тh }}$, то $\mathfrak{B} \in$ Th. Обозначим через $Q_{n}$ множество всех тавтологий, содержащих не более одного символа $f \in \Sigma_{\sigma}$, где $\sigma \in\{\beta, \gamma, \delta\}$, не содержащих символов $f \in \Sigma_{\alpha}$ и содержащих только переменные $\left\{x_{1}, \ldots, x_{n}\right\}$. Тогда несложно убедиться, что найдется $n \in \mathbb{N}$, для которого $\left[Q_{n}\right]_{\Omega_{L}(\Sigma)}=$ Th. Так как для любого $n \in \mathbb{N}$ множество $Q_{n}$ конечно, то лемма доказана.

\section{Монотонные пропозициональные исчисления}

Под монотонными пропозициональными исчислениями будем понимать исчисления, множества формул которых образованы логическими связками из $M \subset P_{2}$. Для монотонных пропозициональных исчислений выполнено следующее достаточное условие конечной порожденности. 
Лемма 12. Если $\Sigma \subseteq M,|\Sigma|<\infty, 1 \in[\Sigma] u \Omega \subseteq O_{\mathrm{Th}},|\Omega|<\infty, \Omega \vdash \Omega_{M}(\Sigma)$, mo исчисление $(\mathrm{Th}, \Omega)$ конечно-порождено.

Доказательство. Согласно лемме 3 достаточно показать, что исчисление $\left(\right.$ Th, $\Omega_{M}(\Sigma)$ ) конечно-порождено. Поскольку $1 \in[\Sigma]$ и $\Sigma \subseteq M$, то существует $f \in \Sigma$, тождественно равная константе 1. Обозначим через $\Sigma_{1}$ множество $\{f \in \Sigma \mid f \equiv 1\}$. Пусть $m-$ максимальная арность функций из $\Sigma_{1}$; положим $Q_{m}=\left\{\mathfrak{F} \in \Phi_{\Sigma}\left(\left\{x_{1}, \ldots, x_{m}\right\}\right) \mid \mathfrak{F}=f x_{i_{1}} \ldots x_{i_{n}}\right.$ и $\left.f \in \Sigma_{1}\right\}$.

Докажем индукцией по глубине формул, что Тh $\subseteq\left[Q_{m}\right]_{\Omega_{M}(\Sigma)}$. Если $\mathfrak{A} \in \mathrm{Th}-$ формула вида $f \mathfrak{A}_{1} \ldots \mathfrak{A}_{n}$, где $f \in \Sigma_{1}$, то $\mathfrak{A} \in\left[Q_{m}\right]_{\Omega_{M}(\Sigma)}$. Если $\mathfrak{A} \in \mathrm{Th}-$ формула вида $f \mathfrak{A}_{1} \ldots \mathfrak{A}_{n}$, где $f \neq \Sigma_{1}$, то рассмотрим сокращенную д.н.ф. функции $f$ :

$$
\bigvee_{i=1}^{k} x_{i_{1}} \& \ldots \& x_{i_{n_{i}}} .
$$

Так как $\mathfrak{A} \in \mathrm{Th}$, то для некоторого конъюнкта $x_{i_{1}} \& \ldots \& x_{i_{n_{i}}}$ имеем $\mathfrak{A}_{i_{1}}, \ldots, \mathfrak{A}_{i_{n_{i}}} \in$ Th. По индуктивному предположению $\mathfrak{A}_{i_{1}}, \ldots, \mathfrak{A}_{i_{n_{i}}} \in\left[Q_{m}\right]_{\Omega_{M}(\Sigma)}$. Применяя правила $\Omega_{M}(\Sigma)$, из $\mathfrak{A}_{i_{1}}, \ldots, \mathfrak{A}_{i_{n_{i}}}$ можно вывести формулу $f \mathfrak{A}_{1} \ldots \mathfrak{A}_{n}$, поэтому $\mathfrak{A} \in\left[Q_{m}\right]_{\Omega_{M}(\Sigma)}$.

Обратное включение $\left[Q_{m}\right]_{\Omega_{M}(\Sigma)} \subseteq$ Тh следует из того, что $Q_{n} \subseteq$ Th и $\Omega_{M}(\Sigma) \subseteq$ $O_{\mathrm{Th}}$. Лемма доказана.

\section{Импликативные пропозициональные исчисления}

Под импликативными пропозициональными исчислениями будем понимать исчисления, множества формул которых образованы логическими связками $\Sigma \subseteq P_{2}$, для которых $[\Sigma] \cap I \neq \varnothing$.

Л. Хенкин [11] показал, что каждое пропозициональное исчисление, содержащее классическую импликацию, конечно-порождено относительно операции modus ponens. Покажем, что это верно и для любого импликативного пропозиционального исчисления.

Предположим, что из $\Sigma \subseteq P_{2}$ выразима импликативная функция $\rightarrow$, т.е. в $\Phi_{\Sigma}$ существует такая формула $\mathfrak{F}\left(x_{1}, \ldots, x_{n}, y\right)$, что $f_{\mathfrak{F}}\left(x_{1}, \ldots, x_{n}, y\right)$ - импликативная функция. В этом случае формулу $\mathfrak{F}\left(x_{1}, \ldots, x_{n}, y\right)$ будем обозначать через $x_{1} \ldots x_{n} \rightarrow y$. Если $x=x_{1}=\ldots=x_{n}$, то для краткости будем писать $x \rightarrow y$ вместо $\underbrace{x \ldots x}_{n} \rightarrow y$.

Рассмотрим исчисление $\mathcal{T}=\left(\right.$ Th, $\left.\Omega_{I}(\rightarrow)\right)$, где $\Omega_{I}(\rightarrow)$ состоит из операции

$$
\frac{x_{1}, \ldots, x_{n}, x_{1} \ldots x_{n} \rightarrow y}{y} \text {. }
$$

Обозначим эту операцию через $\omega_{m p}$ и будем называть ее обобщенной операцией modus ponens.

Формализуем понятие вывода. Пусть $\Gamma \subseteq \Phi_{\Sigma}$. Конечную последовательность формул $\mathfrak{F}_{1}, \ldots, \mathfrak{F}_{l}$, в которой каждая формула $\mathfrak{F}_{i}$ либо является аксиомой, либо принадлежит $\Gamma$, либо получена с помощью обобщенной операции modus ponens из формул данной последовательности, индексы которых меньше $i$, будем называть $\oint о p-$ мальным выводом в Г. При этом $l$ называется длиной вывода. Если формула $\mathfrak{A}$ является последней формулой в такой последовательности, то она называется въводимой в $\Gamma$, в этом случае будем писать $\Gamma \vdash \mathfrak{A}$. Если $\Gamma-$ пустое множество, то формула $\mathfrak{A}$ выводима из аксиом, т.е. является теоремой. В этом случае будем просто писать $\vdash \mathfrak{A}$. 
Без ограничения общности будем считать, что $\Sigma=\{\rightarrow, \varphi\}$, где $\rightarrow-$ импликативная функция $x_{1} x_{2} \rightarrow y$, а $\varphi-$ произвольная $m$-арная функция из $P_{2}$. Рассмотрим следующую систему аксиом:

$$
\begin{aligned}
& \text { 1. } x \rightarrow\left(y_{1} y_{2} \rightarrow x\right), \\
& \text { 2. } x_{1} x_{2} \rightarrow x_{i}, \\
& \text { 3. }\left(x_{1} x_{2} \rightarrow\left(y_{1} y_{2} \rightarrow z\right)\right) \rightarrow\left(\left(x_{1} x_{2} \rightarrow y_{1}\right)\left(x_{1} x_{2} \rightarrow y_{2}\right) \rightarrow\left(x_{1} x_{2} \rightarrow z\right)\right), \\
& \text { 4. } \left.\left(x_{i} \rightarrow y\right) \rightarrow\left(\left(x_{1} x_{2} \rightarrow z\right) \rightarrow y\right) \rightarrow y\right) \text {, где } i \in\{1,2\} .
\end{aligned}
$$

$\mathrm{K}$ этой системе добавим $2^{m}$ аксиом, определяющих значения функции $\varphi$ на всевозможных значениях ее переменных. Пусть $x_{1}, \ldots, x_{m}$ - различные переменные; если $\left(x_{1}^{\prime}, \ldots, x_{m}^{\prime}\right) \in E_{2}^{m}$, то положим $\varphi^{\prime}=\varphi\left(x_{1}^{\prime}, \ldots, x_{m}^{\prime}\right) \in E_{2}$. Пусть $y$ - новая переменная, не встречающаяся в $x_{1}, \ldots, x_{m}$. Если $x_{i}^{\prime}=1$, то через $x_{i}^{*}$ обозначим формулу $\left(x_{i} \rightarrow y\right) \rightarrow y$. Если же $x_{i}^{\prime}=0$, то через $x_{i}^{*}$ обозначим формулу $x_{i} \rightarrow y$. По аналогии, если $\varphi^{\prime}=1$, то через $\varphi^{*}$ обозначим формулу $\left(\varphi\left(x_{1}, \ldots, x_{m}\right) \rightarrow y\right) \rightarrow y$, если же $\varphi^{\prime}=0$, то через $\varphi^{*}$ обозначим формулу $\varphi\left(x_{1}, \ldots, x_{m}\right) \rightarrow y$. Таким образом, к уже определенным аксиомам добавим еще $2^{m}$ аксиом вида

$$
x_{1}^{*} \rightarrow\left(x_{2}^{*} \rightarrow \ldots\left(x_{m}^{*} \rightarrow \varphi^{*}\right)\right) .
$$

В дальнейшем, если встретится формула $\mathfrak{A} \in \Phi_{\Sigma}$ и $x_{1}, \ldots, x_{n}$ - различные переменные в $\mathfrak{A},\left(x_{1}^{\prime}, \ldots, x_{n}^{\prime}\right) \in E_{2}^{n}$ и $\mathfrak{A}^{\prime}=f_{\mathfrak{A}}\left(x_{1}^{\prime}, \ldots, x_{n}^{\prime}\right)$, то через $\mathfrak{A}^{*}$ будем обозначать формулу $(\mathfrak{A} \rightarrow y) \rightarrow y$, если $\mathfrak{A}^{\prime}=1$, и $\mathfrak{A} \rightarrow y$, если $\mathfrak{A}^{\prime}=0$.

Для определенного выше понятия вывода верна так называемая Теорема дедукции [2].

Лемма 13 (Теорема дедукции). Если $Г, \mathfrak{A}_{1}, \mathfrak{A}_{2} \vdash \mathfrak{B}$, mо $\Gamma \vdash \mathfrak{A}_{1} \mathfrak{A}_{2} \rightarrow \mathfrak{B}$ для любъх бормул $\mathfrak{A}_{1}, \mathfrak{A}_{2}$ и $\mathfrak{B}$ и любого множества формул $Г$.

Доказательство. Доказательство будем вести индукцией по длине вывода $\mathfrak{F}_{1}, \ldots, \mathfrak{F}_{n}=\mathfrak{B}$ формулы $\mathfrak{B}$ из $\Gamma, \mathfrak{A}_{1}, \mathfrak{A}_{2}$.

Если $\mathfrak{B}$ - аксиома, либо $\mathfrak{B} \in \Gamma$, то доказательство следует из аксиомы 1 . Если $\mathfrak{B}=\mathfrak{A}_{i}$, то доказательство следует из аксиомы 2.

Пусть $\mathfrak{B}$ - это результат применения обобщенной операции modus ponens к формулам $\mathfrak{F}_{i_{1}}, \mathfrak{F}_{i_{2}}, \mathfrak{F}_{j}$, причем $\mathfrak{F}_{j}$ имеет вид $\mathfrak{F}_{i_{1}} \mathfrak{F}_{i_{2}} \rightarrow \mathfrak{B}$. По предположению индукции

$$
\begin{aligned}
\Gamma \vdash \mathfrak{A}_{1} \mathfrak{A}_{2} \rightarrow \mathfrak{F}_{i_{1}}, \\
\Gamma \vdash \mathfrak{A}_{1} \mathfrak{A}_{2} \rightarrow \mathfrak{F}_{i_{2}}, \\
\Gamma \vdash \mathfrak{A}_{1} \mathfrak{A}_{2} \rightarrow\left(\mathfrak{F}_{i_{1}} \mathfrak{F}_{i_{2}} \rightarrow \mathfrak{B}\right) .
\end{aligned}
$$

Объединим выводы этих формул и добавим к ним следующие три формулы:

$$
\begin{gathered}
\left(\mathfrak{A}_{1} \mathfrak{A}_{2} \rightarrow\left(\mathfrak{F}_{i_{1}} \mathfrak{F}_{i_{2}} \rightarrow \mathfrak{B}\right)\right) \rightarrow\left(\left(\mathfrak{A}_{1} \mathfrak{A}_{2} \rightarrow \mathfrak{F}_{i_{1}}\right)\left(\mathfrak{A}_{1} \mathfrak{A}_{2} \rightarrow \mathfrak{F}_{i_{2}}\right) \rightarrow\left(\mathfrak{A}_{1} \mathfrak{A}_{2} \rightarrow \mathfrak{B}\right)\right), \\
\left(\mathfrak{A}_{1} \mathfrak{A}_{2} \rightarrow \mathfrak{F}_{i_{1}}\right)\left(\mathfrak{A}_{1} \mathfrak{A}_{2} \rightarrow \mathfrak{F}_{i_{2}}\right) \rightarrow\left(\mathfrak{A}_{1} \mathfrak{A}_{2} \rightarrow \mathfrak{B}\right), \\
\mathfrak{A}_{1} \mathfrak{A}_{2} \rightarrow \mathfrak{B} .
\end{gathered}
$$

Первая получена из аксиомы 3, две последние выводятся из первой с помощью обобщенной операции modus ponens. Образованная последовательность формул является выводом формулы $\mathfrak{A}_{1} \mathfrak{A}_{2} \rightarrow \mathfrak{B}$ из $Г$. 
Лемма 14. Пусть $\mathfrak{A} \in \Phi_{\Sigma} u x_{1}, \ldots, x_{n}-$ различные переменные, встречающиеся в $\mathfrak{A}$. Пусть $\left(x_{1}^{\prime}, \ldots, x_{n}^{\prime}\right) \in E_{2}^{n}$ u $\mathfrak{A}^{\prime}=f_{\mathfrak{A}}\left(x_{1}^{\prime}, \ldots, x_{n}^{\prime}\right)$. Тогда

$$
x_{1}^{*}, \ldots, x_{n}^{*} \vdash \mathfrak{A}^{*} .
$$

Доказательство. Будем доказывать индукцией по длине формулы $\mathfrak{A}$.

Если $\mathfrak{A}$ - переменная $x_{i}$, то утверждение леммы следует из определения выводимости $\vdash$.

Предположим, что утверждение леммы верно для любой формулы глубины $l^{\prime}, l^{\prime}<l$, докажем его для формулы $\mathfrak{A}$ глубины $l$. Если $\mathfrak{A}$ - это формула $\varphi\left(\mathfrak{A}_{1}, \ldots, \mathfrak{A}_{m}\right)$, то по индуктивному предположению утверждение леммы верно для формул $\mathfrak{A}_{1}, \ldots, \mathfrak{A}_{m}$, т.е.

$$
x_{1}^{*}, \ldots, x_{n}^{*} \vdash \mathfrak{A}_{i}^{*}, i=1, \ldots, m \text {. }
$$

Кроме того, из аксиомы для логической связки $\varphi$ имеем

$$
\mathfrak{A}_{1}^{*}, \ldots, \mathfrak{A}_{m}^{*} \vdash \mathfrak{A}^{*}
$$

Следовательно,

$$
x_{1}^{*}, \ldots, x_{n}^{*} \vdash \mathfrak{A}^{*} .
$$

Пусть $\mathfrak{A}$ - это формула $\mathfrak{B}_{1} \mathfrak{B}_{2} \rightarrow \mathfrak{C}$ и утверждение леммы верно для формул $\mathfrak{B}_{1}, \mathfrak{B}_{2}$ и $\mathfrak{C}:$

$$
\begin{aligned}
& x_{1}^{*}, \ldots, x_{n}^{*} \vdash \mathfrak{B}_{i}^{*}, \\
& x_{1}^{*}, \ldots, x_{n}^{*} \vdash \mathfrak{C}^{*} .
\end{aligned}
$$

Возможны следующие случаи:

1) Если $\mathfrak{B}_{i}^{\prime}=0$, то $\mathfrak{A}^{\prime}=1$. Тогда $\mathfrak{B}_{i}^{*}=\mathfrak{B}_{i} \rightarrow y, \mathfrak{A}^{*}=(\mathfrak{A} \rightarrow y) \rightarrow y$ и по аксиоме 4 имеем

$$
\left.\vdash\left(\mathfrak{B}_{i} \rightarrow y\right) \rightarrow\left(\left(\mathfrak{B}_{1} \mathfrak{B}_{2} \rightarrow \mathfrak{C}\right) \rightarrow y\right) \rightarrow y\right)
$$

2) Если $\mathfrak{C}^{\prime}=1$, то $\mathfrak{A}^{\prime}=1$. Тогда $\mathfrak{C}^{*}=(\mathfrak{C} \rightarrow y) \rightarrow y$ и $\mathfrak{A}^{*}=(\mathfrak{A} \rightarrow y) \rightarrow y$. Имеют место следующие соотношения:

$$
\begin{aligned}
& \mathfrak{C} \vdash \mathfrak{B}_{1} \mathfrak{B}_{2} \rightarrow \mathfrak{C}, \\
& \mathfrak{C},\left(\mathfrak{B}_{1} \mathfrak{B}_{2} \rightarrow \mathfrak{C}\right) \rightarrow y \vdash y,\left.\rightarrow \mathfrak{B}_{1} \mathfrak{B}_{2} \rightarrow \mathfrak{C}\right) \rightarrow y \vdash \mathfrak{C} \rightarrow y, \\
&\left(\mathfrak{B}_{1} \mathfrak{B}_{2} \rightarrow \mathfrak{C}\right) \rightarrow y,(\mathfrak{C} \rightarrow y) \rightarrow y \vdash y, \\
&(\mathfrak{C} \rightarrow y) \rightarrow y \vdash\left(\left(\mathfrak{B}_{1} \mathfrak{B}_{2} \rightarrow \mathfrak{C}\right) \rightarrow y\right) \rightarrow y,
\end{aligned}
$$

где первый вывод есть использование аксиомы 1, второй и четвертый - применение modus ponens, остальные получены по теореме дедукции.

3) Если $\mathfrak{B}_{1}^{\prime}, \mathfrak{B}_{2}^{\prime}=1, \mathfrak{C}^{\prime}=0$, то $\mathfrak{A}^{\prime}=0$. Тогда $\mathfrak{B}_{i}^{*}=\left(\mathfrak{B}_{i} \rightarrow y\right) \rightarrow y, \mathfrak{C}^{*}=\mathfrak{C} \rightarrow y$ и 
$\mathfrak{A}^{*}=\mathfrak{A} \rightarrow y$. Имеют место следующие соотношения:

$$
\begin{aligned}
\mathfrak{B}_{1}, \mathfrak{B}_{2}, \mathfrak{B}_{1} \mathfrak{B}_{2} \rightarrow \mathfrak{C} \vdash \mathfrak{C}, \\
\mathfrak{B}_{1}, \mathfrak{B}_{2}, \mathfrak{B}_{1} \mathfrak{B}_{2} \rightarrow \mathfrak{C}, \mathfrak{C}^{*} \vdash y, \\
\mathfrak{B}_{2}, \mathfrak{B}_{1} \mathfrak{B}_{2} \rightarrow \mathfrak{C}, \mathfrak{C}^{*} \vdash \mathfrak{B}_{1} \rightarrow y, \\
\mathfrak{B}_{1}^{*}, \mathfrak{B}_{2}, \mathfrak{B}_{1} \mathfrak{B}_{2} \rightarrow \mathfrak{C}, \mathfrak{C}^{*} \vdash y, \\
\mathfrak{B}_{1}^{*}, \mathfrak{B}_{1} \mathfrak{B}_{2} \rightarrow \mathfrak{C}, \mathfrak{C}^{*} \vdash \mathfrak{B}_{2} \rightarrow y, \\
\mathfrak{B}_{1}^{*}, \mathfrak{B}_{2}^{*}, \mathfrak{B}_{1} \mathfrak{B}_{2} \rightarrow \mathfrak{C}, \mathfrak{C}^{*} \vdash y, \\
\mathfrak{B}_{1}^{*}, \mathfrak{B}_{2}^{*}, \mathfrak{C}^{*} \vdash\left(\mathfrak{B}_{1} \mathfrak{B}_{2} \rightarrow \mathfrak{C}\right) \rightarrow y,
\end{aligned}
$$

где первый, второй, четвертый и шестой выводы являются применением modus ponens, остальные получены по теореме дедукции.

Для каждого случая из (2) и (3) следует

$$
x_{1}^{*}, \ldots, x_{n}^{*} \vdash \mathfrak{A}^{*} .
$$

Разбор всех случаев завершает доказательство леммы.

Лемма 15 (Непротиворечивости). Любая формула $\mathfrak{A} \in \Phi_{\Sigma}$ является тавтологией, если $\vdash \mathfrak{A}$.

Доказательство. Действительно, каждая аксиома является тавтологией, правила вывода, будучи примененными к тавтологиям, выводят тавтологии. Лемма доказана.

Докажем теперь обратную лемму.

Лемма 16 (Полноты). Если формула $\mathfrak{A} \in \Phi_{\Sigma}$ является тавтологией, то $\vdash \mathfrak{A}$.

Доказательство. Пусть $x_{1}, \ldots, x_{n}$ - различные переменные, встречающиеся в $\mathfrak{A}$. Так как $\mathfrak{A}$ - тавтология, то для любого набора $\left(x_{1}^{\prime}, \ldots, x_{n}^{\prime}\right) \in E_{2}$ значение $\mathfrak{A}^{\prime}=$ $f_{\mathfrak{A}}\left(x_{1}^{\prime}, \ldots, x_{n}^{\prime}\right)=1$. По лемме 14 для каждого из $2^{n}$ возможных множеств $\Gamma_{n}=$ $\left\{x_{1}^{*}, \ldots, x_{n}^{*}\right\}$ имеем

$$
\Gamma_{n} \vdash(\mathfrak{A} \rightarrow y) \rightarrow y .
$$

Это означает, что для каждого из $2^{n-1}$ возможных множеств $\Gamma_{n-1}=\left\{x_{1}^{*}, \ldots, x_{n-1}^{*}\right\}$ верно

$$
\begin{aligned}
\Gamma_{n-1}, x_{n} & \rightarrow y \vdash(\mathfrak{A} \rightarrow y) \rightarrow y, \\
\Gamma_{n-1}, \quad\left(x_{n} \rightarrow y\right) & \rightarrow y \vdash(\mathfrak{A} \rightarrow y) \rightarrow y .
\end{aligned}
$$

Отсюда по Теореме дедукции

$$
\begin{aligned}
& \Gamma_{n-1} \vdash\left(x_{n} \rightarrow y\right) \rightarrow(\mathfrak{A} \rightarrow y) \rightarrow y, \\
& \Gamma_{n-1} \vdash\left(\left(x_{n} \rightarrow y\right) \rightarrow y\right) \rightarrow(\mathfrak{A} \rightarrow y) \rightarrow y .
\end{aligned}
$$

Поскольку тавтология

$$
\begin{aligned}
\left(\left(x_{n} \rightarrow y\right) \rightarrow((\mathfrak{A} \rightarrow y)\right. & \rightarrow y)) \rightarrow \\
& \rightarrow\left(\left(\left(\left(x_{n} \rightarrow y\right) \rightarrow y\right) \rightarrow((\mathfrak{A} \rightarrow y) \rightarrow y)\right) \rightarrow((\mathfrak{A} \rightarrow y) \rightarrow y)\right)
\end{aligned}
$$


выводима из аксиомы 4, то с помощью двукратного применения операции modus ponens получим

$$
\vdash(\mathfrak{A} \rightarrow y) \rightarrow y
$$

Подставляя вместо y формулу $\mathfrak{A}$, имеем

$$
\vdash(\mathfrak{A} \rightarrow \mathfrak{A}) \rightarrow \mathfrak{A} .
$$

Так как $\mathfrak{A} \vdash \mathfrak{A}$, то по Теореме дедукции $\vdash \mathfrak{A} \rightarrow \mathfrak{A}$. Тогда, применяя операцию modus ponens, окончательно получаем, что

$\vdash \mathfrak{A}$.

Лемма доказана.

Для импликативных пропозициональных исчислений имеет место следующее достаточное условие конечной порожденности.

Лемма 17. Если $\Sigma \subseteq P_{2},|\Sigma|<\infty, \Omega \subseteq O_{\mathrm{Th}},|\Omega|<\infty$, и найдется такая функиия $f \in[\Sigma] \cap I$, что $\Omega \vdash \Omega_{I}(f)$, то исчисление $(\mathrm{Th}, \Omega)$ конечно-порождено.

Доказательство. Согласно лемме 3 достаточно показать, что исчисление $\left(\mathrm{Th}, \Omega_{I}(f)\right)$ конечно-порождено. Согласно леммам 15 и 16 система аксиом 1-4 и аксиом для символа $\varphi$ порождает все множество тавтологий Тh и только их. Поскольку эта система конечна, исчисление $(\mathrm{Th}, \Omega)$ конечно-порождено. Лемма доказана.

\section{Доказательство теоремы 1}

Необходимость условий следует из леммы 2. Достаточность условия 1) следует из леммы 11, достаточность условия 2) следует из леммы 12 , достаточность условия 3 ) следует из леммы 17. Достаточность условий 4) следует из того, что Th порождается конечным множеством тавтологий.

\section{Существование базиса сколь угодно большой конечной мощ- ности}

Лемма 18. Для любого $n \geqslant 12$ существует полная независимая система тавтологий в (Th, $\left.\left\{\omega_{m p}\right\}\right)$ мощности $n$.

Доказательство. Рассмотрим следующую полную независимую систему тавтоло- 
гий $M$ :

$$
\begin{aligned}
\text { 1) } & x \rightarrow(y \rightarrow x), \\
2) & (x \rightarrow(x \rightarrow y)) \rightarrow(x \rightarrow y), \\
3) & (x \rightarrow y) \rightarrow((y \rightarrow z) \rightarrow(x \rightarrow z)), \\
4) & x \wedge y \rightarrow x, \\
5) & x \wedge y \rightarrow y, \\
6) & (x \rightarrow y) \rightarrow((x \rightarrow z) \rightarrow(x \rightarrow y \wedge z)), \\
7) & x \rightarrow x \vee y, \\
8) & y \rightarrow x \vee y, \\
9) & (x \rightarrow z) \rightarrow((y \rightarrow z) \rightarrow(x \vee y \rightarrow z)), \\
10) & (x \rightarrow y) \rightarrow(\bar{y} \rightarrow \bar{x}), \\
11) & \bar{x} \rightarrow x \\
12) & x \rightarrow \overline{\bar{x}}
\end{aligned}
$$

Обозначим формулы данной системы через $\mathfrak{A}_{1}, \ldots, \mathfrak{A}_{12}$ соответственно и положим

$$
\begin{aligned}
\mathfrak{B}_{0} & =\mathfrak{A}_{1} \vee \ldots \vee \mathfrak{A}_{12}, \\
\mathfrak{B}_{i+1} & =\overline{\overline{B_{i}}}, i=0, \ldots, n-1, \\
\mathfrak{C}_{i} & =\mathfrak{B}_{0} \rightarrow \mathfrak{A}_{i}, i=1, \ldots, 11, \\
\mathfrak{C}_{12} & =\mathfrak{A}_{12}, \\
\mathfrak{D}_{i} & =\mathfrak{B}_{i+1} \rightarrow \mathfrak{B}_{i}, i=0, \ldots, n-1, \\
\mathfrak{E} & =(x \rightarrow x) \rightarrow \overline{\overline{x \rightarrow x}}, \\
\mathfrak{D}_{n} & =\mathfrak{E} \rightarrow \mathfrak{B}_{n} .
\end{aligned}
$$

Докажем, что для любого $n \geqslant 0$ система

$$
M_{n}=\left\{\mathfrak{C}_{1}, \ldots, \mathfrak{C}_{12}, \mathfrak{D}_{0}, \ldots, \mathfrak{D}_{n}\right\}
$$

является базисом в Тh мощности $n+13$.

Непосредственно из определения системы $M_{n}$ следует, что для любого $n \geqslant 0$ выполнено включение $M \subseteq\left[M_{n}\right]$. Так как $[M]=\mathrm{Th}$, то система $M_{n}$ является полной в Th для любого $n \geqslant 0$.

Покажем, что для каждого $n \geqslant 0$ система $M_{n}$ является базисом. Для этого воспользуемся методом оценок (см. [1] стр. 103). Обозначим через $P_{k}$ функции $k$ значной логики. Оценкой называется пара $(\theta, Q)$, где $\theta$ - это отображение

$$
\theta:\{\wedge, \vee, \neg, \rightarrow\} \rightarrow P_{k},
$$

сопоставляющее логическим связкам $\wedge, \vee, \neg, \rightarrow$ функции $\theta_{\wedge}, \theta_{\vee}, \theta_{\neg}, \theta_{\rightarrow}$ из $P_{k}$ той же арности, а $Q$ - это выделенное подмножество $E_{k}=\{0, \ldots, k-1\}$. Каждой формуле $\mathfrak{F} \in \Phi_{\Sigma}$ однозначно соответствует функция из $\left[\left\{\theta_{\wedge}, \theta_{\vee}, \theta_{\neg}, \theta_{\rightarrow}, g(x)\right\}\right]$, где $g(x) \in P_{k}$ - тождественная функция. При этом каждой переменной соответствует тождественная функция; если формулам $\mathfrak{A}_{1}, \mathfrak{A}_{2}$ соответствуют функции $f_{1}, f_{2}$, то формулам $\mathfrak{A}_{1} \wedge \mathfrak{A}_{2}, \mathfrak{A}_{1} \vee \mathfrak{A}_{2}, \overline{\mathfrak{A}_{1}}, \mathfrak{A}_{1} \rightarrow \mathfrak{A}_{2}$ соответствуют функции $\theta_{\wedge}\left(f_{1}, f_{2}\right), \theta_{\vee}\left(f_{1}, f_{2}\right), \theta_{\neg}\left(f_{1}\right), \theta_{\rightarrow}\left(f_{1}, f_{2}\right)$ соответственно. Итак, каждой формуле $\mathfrak{F}$ из $\Phi_{\Sigma}$ можно сопоставить функцию из $P_{k}$, которую будем обозначать через $\theta_{\mathfrak{F}}$. 
Метод оценок состоит в следующем. Чтобы доказать, что некоторая формула $\mathfrak{B}$ не выводится из формул $\mathfrak{A}_{1}, \ldots, \mathfrak{A}_{n}$, достаточно указать такую оценку $(\theta, Q)$, что функции $\theta_{\mathfrak{A}_{1}}, \ldots, \theta_{\mathfrak{A}_{n}}$ принимают только выделенные значения из $Q$ и для любых формул $\mathfrak{C}$ и $\mathfrak{C} \rightarrow \mathfrak{D}$, функции $\theta_{\mathfrak{C}}$ и $\theta_{\mathfrak{C} \rightarrow \mathfrak{D}}$ которых принимают только выделенные значения из $Q$, тем же свойством обладает и функция $\theta_{\mathfrak{D}}$, а функция $\theta_{\mathfrak{B}}$, напротив, принимает также и невыделенные значения.

Для каждой тавтологии $\mathfrak{A}_{i}$ из системы $M$ существует оценка $\left(\theta^{i}, Q^{i}\right)$, в соответствии с которой доказывается ее независимость в данной системе (см. [1] стр. 107). Нетрудно убедиться, что для любого $n \geqslant 0$ и каждого $i, 1 \leqslant i \leqslant 12$, пара $\left(\theta^{i}, Q^{i}\right)$ является оценкой, доказывающей независимость формулы $\mathfrak{C}_{i}$ в системе $M_{n}$. В самом деле,

$$
\begin{aligned}
\theta_{\mathfrak{B}_{0}}^{i} & \equiv 1, \text { если } 1 \leqslant i \leqslant 12, \\
\theta_{\mathfrak{B}_{j}}^{i} & \equiv 1, \text { если } 1 \leqslant i \leqslant 11 \text { и } 1 \leqslant j \leqslant n, \\
\theta_{\mathfrak{B}_{j}}^{i} & \equiv 0, \text { если } i=12 \text { и } 1 \leqslant j \leqslant n, \\
\theta_{\mathfrak{E}}^{i} & \equiv 1, \text { если } 1 \leqslant i \leqslant 11, \\
\theta_{\mathfrak{E}}^{i} & \equiv 0, \text { если } i=12 .
\end{aligned}
$$

Поэтому для любого $i, 1 \leqslant i \leqslant 12, \theta_{\mathfrak{C}_{j}}^{i} \equiv 1$ тогда и только тогда, когда $j \neq i$. Кроме того, $\theta_{\mathfrak{D}_{j}}^{i} \equiv 1$ для любых $i=1, \ldots, 12$, и $j=0, \ldots, n$. Следовательно, формула $\mathfrak{C}_{i}$ не выводима из $M_{n} \backslash\left\{\mathfrak{C}_{i}\right\}$ для любого $i=1, \ldots, 12$. Остается построить оценку $\left(\eta^{i}, R^{i}\right), i=0, \ldots, n$, доказывающую независимость формулы $\mathfrak{D}_{i}$. 1),

Для $i=0, \ldots, n$ обозначим через $\eta_{\wedge}^{i}$ произвольную функцию из $P_{2 i+3}, \eta_{\vee}^{i} \equiv 2(i+$

$$
\eta_{\neg}^{i}(x)=\left\{\begin{array}{c}
x-1, \text { если } 3 \leqslant x \leqslant 2(i+1), \\
0, \text { если } 1 \leqslant x \leqslant 2, \\
1, \text { если } x=0 .
\end{array}\right.
$$

Функция $\eta_{\rightarrow}^{i}$ удовлетворяет условиям:

$$
\begin{aligned}
\eta_{\rightarrow}^{i}(x, x) & \equiv 1, \forall x \in E_{2 i+3}, \\
\eta_{\rightarrow}^{i}(1, x) & \equiv 0, \forall x \in E_{2 i+3} \backslash\{1\}, \\
\eta_{\rightarrow}^{i}(x, 1) & \equiv 1, \forall x \in E_{2 i+3}, \\
\eta_{\rightarrow \rightarrow}^{i}(2(i+1), x) & \equiv 1, \forall x \in E_{2 i+3}, \\
\eta_{\rightarrow}^{i}(2(j-1), 2 j) & =1, \quad j=2, \ldots, i+1, \\
\eta_{\rightarrow}^{i}(2 j, 2(j-1)) & =1, \quad j=2, \ldots, i+1,
\end{aligned}
$$

а на остальных наборах $(x, y) \in E_{2 i+3}^{2}$ значение функции $\eta_{\rightarrow}^{i}(x, y)=0$. 
Для любой оценки $\left(\eta^{i},\{1\}\right), i=0, \ldots, n$, выполнено

$$
\begin{aligned}
\eta_{\mathfrak{B}_{0}}^{i} \equiv 2(i+1) & \Rightarrow \eta_{\mathfrak{C}_{j}}^{i} \equiv 1, j=1, \ldots, 11, \\
\eta_{\mathfrak{B}_{1}}^{i} \equiv 2 i \Rightarrow \eta_{\mathfrak{D}_{0}}^{i} \equiv 1, & \quad \cdots \\
\eta_{\mathfrak{B}_{i}}^{i} \equiv 2 & \Rightarrow \eta_{\mathfrak{D}_{i-1}}^{i} \equiv 1, \\
\eta_{\mathfrak{B}_{i+1}}^{i} \equiv 1 & \Rightarrow \eta_{\mathfrak{D}_{i}}^{i} \equiv 0 \\
\eta_{\mathfrak{B}_{i+2}}^{i} \equiv 1 & \Rightarrow \eta_{\mathfrak{D}_{i+1}}^{i} \equiv 1, \\
& \quad \cdots \\
\eta_{\mathfrak{B}_{n}}^{i} \equiv 1 & \Rightarrow \eta_{\mathfrak{D}_{n-1}}^{i} \equiv 1, \\
\eta_{\mathfrak{E}}^{i} \equiv 1 & \Rightarrow \eta_{\mathfrak{D}_{n}}^{i} \equiv 1, \\
\eta_{\mathfrak{C}_{12}}^{i} \equiv 1 . &
\end{aligned}
$$

Отсюда следует, что формула $\mathfrak{D}_{i}$ не выводима из $M_{n} \backslash\left\{\mathfrak{D}_{i}\right\}$. Следовательно, система $M_{n}$ является базисом.

Итак, для любого $n \geqslant 12$ в Тh существует базис мощности $n$. Лемма доказана.

\section{Существование счетного базиса}

Рассмотрим множество тавтологий

$$
M=\{\mathfrak{A}_{k} \mid \mathfrak{A}_{k}=\underbrace{(x \vee \bar{x}) \wedge \ldots \wedge(x \vee \bar{x})}_{k}, k \geqslant 1\} .
$$

Лемма 19. Замкнутый класс тавтологий $[M]_{\omega_{m p}}$ имеет счетный базис.

Доказательство. Покажем, что множество $M$ является базисом в $[M]$. В самом деле, к формулам из множества $M$ можно применить только правило подстановки, которое не изменяет числа сомножителей в конъюнкции. Поэтому для любого $k \geqslant 1$ формулу $\mathfrak{A}_{k}$ нельзя вывести из $M \backslash\left\{\mathfrak{A}_{k}\right\}$. Лемма доказана.

\section{Существование класса без базиса}

Обозначим через $\mathfrak{A}_{k}$ тавтологию

$$
\underbrace{(x \vee \bar{x}) \wedge \ldots \wedge(x \vee \bar{x})}_{k}
$$

где $k \geqslant 1$. Положим для $k \geqslant 1$ и $n \geqslant 0$

$$
\mathfrak{B}_{k, n}=\mathfrak{A}_{k+n} \rightarrow\left(\mathfrak{A}_{k+n-1} \rightarrow \ldots \rightarrow\left(\mathfrak{A}_{k+1} \rightarrow \mathfrak{A}_{k}\right)\right) .
$$

Для тавтологий $\mathfrak{B}_{k, n}$ выполнено рекуррентное соотношение

$$
\mathfrak{B}_{k, n+1}=\mathfrak{B}_{k+n+1,0} \rightarrow \mathfrak{B}_{k, n} .
$$

Для множества тавтологий $M=\left\{\mathfrak{B}_{k, n} \mid k \geqslant 1, n \geqslant 0\right\}$ верна следующая лемма.

Лемма 20. Замкнутый класс тавтологий $[M]_{\omega_{m p}}$ не имеет базиса. 
Доказательство. Покажем, что в замыкании $[M]_{\omega_{m p}}$ содержатся лишь подстановочные варианты формул множества $M$. Доказывать будем индукцией по числу применений операции modus ponens, поскольку операция подстановки сохраняет это свойство. Обозначим через $M_{l}$ множество тавтологий, выводимых из $M$ не более чем за $l$ применений операции modus ponens. Тогда для $M_{0}$ утверждение верно. Пусть оно верно для $M_{l}$, докажем его для $M_{l+1}$. Пусть тавтология $\mathfrak{A} \in M_{l+1}$ выводима из $\mathfrak{B}, \mathfrak{C} \in M_{l}$. Согласно (4) тавтологии $\mathfrak{A}, \mathfrak{B}, \mathfrak{C}$ имеют вид $\mathfrak{B}_{k, n}^{\prime}, \mathfrak{B}_{k, n+1}^{\prime}, \mathfrak{B}_{k+n+1,0}^{\prime}$, где штрихи означают подстановочные варианты соответствующих формул. Утверждение доказано.

Предположим противное, что $[M]_{\omega_{m p}}$ содержит базис $M^{\prime}$. Обозначим через $M_{k, n}^{\prime}$ наименьшее подмножество в $M^{\prime}$, из которого выводима формула $\mathfrak{B}_{k, n}$. Поскольку $M^{\prime}$ является базисом, для каждого $k \geqslant 1$ и $n \geqslant 0$ множество $M_{k, n}^{\prime}$ не пусто. Несложно заметить, что $\mathfrak{B}_{k, n}$ выводима из $M_{k, n}^{\prime}$ только с помощью применения операции modus ponens. Докажем индукцией по числу $l$ применений операции modus ponens, что $M_{k, n}^{\prime} \subseteq M$ и для любого $\mathfrak{B}_{k^{\prime}, n^{\prime}} \in M_{k, n}^{\prime}$ выполнено $k^{\prime}+n^{\prime} \geqslant k+n$.

Для $l=0$ верно включение $\mathfrak{B}_{k, n} \in M_{k, n}^{\prime}$, следовательно, $M_{k, n}^{\prime}=\left\{\mathfrak{B}_{k, n}\right\}$ и утверждение верно.

Пусть утверждение верно для всех $l^{\prime}<l$, докажем его для $l$. Согласно (4) формула $\mathfrak{B}_{k, n}$ может быть выведена только из формул $\mathfrak{B}_{k, n+1}$ и $\mathfrak{B}_{k+n+1,0}$. Тогда $\mathfrak{B}_{k, n+1} \in\left[M_{k, n+1}^{\prime}\right], \mathfrak{B}_{k+n+1,0} \in\left[M_{k+n+1,0}^{\prime}\right]$ и $M_{k, n}^{\prime} \subseteq M_{k, n+1}^{\prime} \cup M_{k+n+1,0}^{\prime}$. По предположению индукции $M_{k, n+1}^{\prime}, M_{k+n+1,0}^{\prime} \subseteq M$, поэтому $M_{k, n}^{\prime} \subseteq M$. Если $\mathfrak{B}_{k^{\prime}, n^{\prime}} \in M_{k, n}^{\prime}$, то либо $\mathfrak{B}_{k^{\prime}, n^{\prime}} \in M_{k, n+1}^{\prime}$ и $k^{\prime}+n^{\prime} \geqslant k+n+1>k+n$, либо $\mathfrak{B}_{k^{\prime}, n^{\prime}} \in M_{k+n+1,0}^{\prime}$ и $k^{\prime}+n^{\prime} \geqslant k+n+1>k+n$. В любом случае $k^{\prime}+n^{\prime} \geqslant k+n$. Утверждение доказано.

Из доказанного, в частности, следует, что $M \cap M^{\prime}$ не пусто. Рассмотрим произвольную формулу $\mathfrak{B}_{k, n} \in M \cap M^{\prime}$. Так как $k+n<k+n+1$, то $\mathfrak{B}_{k, n} \notin$ $M_{k, n+1}^{\prime} \cup M_{k+n+1,0}^{\prime}$. Ho $\mathfrak{B}_{k, n} \in\left[\left\{\mathfrak{B}_{k, n+1}, \mathfrak{B}_{k+n+1,0}\right\}\right]_{\omega_{m p}} \subseteq\left[M_{k, n+1}^{\prime} \cup M_{k+n+1,0}^{\prime}\right]_{\omega_{m p}} \subseteq$ $\left[M^{\prime} \backslash\left\{\mathfrak{B}_{n, k}\right\}\right]_{\omega_{m p}}$, поэтому $M^{\prime}$ не является базисом. Лемма доказана.

\section{Существование класса с базисом, содержащего полную подси- стему без базиса}

Рассмотрим множество тавтологий $M=\left\{\mathfrak{A}_{i} \mid i \geqslant 0\right\}$, где

$$
\begin{gathered}
\mathfrak{A}_{0}=x \rightarrow x, \\
\mathfrak{A}_{i}=\mathfrak{A}_{i-1} \rightarrow \ldots \rightarrow\left(\mathfrak{A}_{0} \rightarrow\left(x_{i} \rightarrow \ldots \rightarrow\left(x_{1} \rightarrow \mathfrak{A}_{0}\right)\right)\right), i \geqslant 1 .
\end{gathered}
$$

Лемма 21. Множество тавтологий $M$ является базисом замкнутого класса $[M]_{\omega_{m p}}$.

Доказательство. Предположим противное: существует такое $i \geqslant 0$, что $\mathfrak{A}_{i}$ выводима из $M \backslash\left\{\mathfrak{A}_{i}\right\}$. Рассмотрим наименьший вывод $\Gamma$ этой формулы. Поскольку $\mathfrak{A}_{i}$ содержится в посылках каждой тавтологии $\mathfrak{A}_{i^{\prime}}, i^{\prime}>i$, то формулы $\mathfrak{A}_{i^{\prime}}$ для $i^{\prime}>i$ не участвуют в выводе $\Gamma$. Далее, каждая формула $\mathfrak{A}_{i^{\prime}}$ для $i^{\prime}<i$ имеет не более $2 i$ посылок, а операции подстановки и modus ponens, будучи примененными к тавтологиям данного вида, не могут привести к формуле того же вида, число посылок которой превосходит $2 i$. Но тавтология $\mathfrak{A}_{i}$ имеет $2 i+1$ посылок, получили противоречие. Лемма доказана.

Лемма 22. Замкнутвй класс тавтологий $[M]_{\omega_{m p}}$ содержит полую подсистему, из которой нельзя выделить базис. 
Доказательство. Рассмотрим множество тавтологий $M^{\prime}=\left\{\mathfrak{B}_{i} \mid \mathfrak{B}_{i}=x_{i} \rightarrow \ldots \rightarrow\right.$ $\left.\left(x_{1} \rightarrow \mathfrak{A}_{0}\right), i \geqslant 0\right\}$. Ясно, что $\left[M^{\prime}\right]_{\omega_{m p}}=[M]_{\omega_{m p}}$, значит, множество $M^{\prime}$ является полным. Покажем, что из него нельзя выделить базис.

Множество $M^{\prime}$ обладает тем свойством, что из тавтологий $\mathfrak{B}_{i}$ с индексами $i<m$ нельзя вывести тавтологии $\mathfrak{B}_{m}$, поэтому любое конечное подмножество $M^{\prime}$ не является полным в $M$. С другой стороны, из тавтологии $\mathfrak{B}_{m}$ выводима любая тавтология $\mathfrak{B}_{i}$ с меньшим индексом, поэтому из любой полной подсистемы в $M^{\prime}$ всегда можно убрать тавтологии с меньшими номерами. Следовательно, из $M^{\prime}$ нельзя выделить базис. Лемма доказана.

\section{Доказательство теоремы 2}

Доказательство первого утверждения следует из леммы 18, второго - из леммы 19, третьего - из леммы 20 и четвертого - из леммы 22.

\section{Список литературы}

1. Гильберт Д., Бернайс П., Логические исчисления и формализачия арифметики. Наука, Москва, 1979.

2. Колмогоров А. Н., Драгалин А. Г., Введение в математическую логику. Изд-во Моск. ун-та, Москва, 1982.

3. Кон П., Универсалъная алгебра. Мир, Москва, 1968.

4. Минц Г. Е., Допустимые и производные правила. Записки научных семинаров ЛОМИ AH CCCP (1968) 8, 189-191.

5. Новиков П. С., Элементы математической логики. Наука, Москва, 1973.

6. Циткин А. И., О допустимых правилах интуиционистской логики высказываний. Матем. сб. (1977) 102(144), №2, 314-323.

7. Шенфилд Д., Математическая логика. Наука, Москва, 1975.

8. Яблонский С. В., Введение в дискретную математику. Наука, М, 1986.

9. Яблонский С. В., Гаврилов Г. П., Кудрявцев В. Б., Функиии алгебры логики и классъ Поста. Наука, Москва, 1966.

10. Янов Ю. И., Мучник А. А., О существовании $k$-значных замкнутых классов, не имеющих конечного базиса. ДАН СССР (1959) 127, №1, 44-46.

11. Henkin L., Fragments of the propositional calculus. J. Symb.Logic (1949) 14, 42-82.

12. Post E., Two-valued iterative systems of mathematical logic. Princeton, 1941.

Статья поступила 07.06.2013. 